\title{
A new LC-MS/MS method for multiple residues/contaminants in bovine meat
}

\author{
Zehra Hajrulai-Musliu ${ }^{1 *}$, Risto Uzunov ${ }^{1}$, Stefan Jovanov ${ }^{1}$, Dean Jankuloski ${ }^{1}$, Velimir Stojkovski ${ }^{1}$, \\ Lazo Pendovski and James Jacob Sasanya ${ }^{2}$
}

\begin{abstract}
A multi-class and multi-residue/contaminant method for the determination of veterinary drug and pesticide residues and mycotoxins in bovine meat has been developed and validated. The veterinary drug residues/contaminants included antimicrobials, anabolic hormones, lactones, $\beta$-agonists, mycotoxins, and pesticides. Isotopic labeled internal standards were included to compensate residual matrix effects. The calibrators used in the method demonstrated linearity with the $R^{2}>0.98$. The decision limit (CCa) values were in the range from 0.067 to $2103.84 \mu \mathrm{g} / \mathrm{kg}$, while the range for detection capability (CC $\beta$ ) was from 0.083 to $2482.13 \mu \mathrm{g} / \mathrm{kg}$. The limit of detection (LOD) and limit of quantification (LOQ) were in the range from 0.059 to $291.36 \mu \mathrm{g} / \mathrm{kg}$, and 0.081 to $328.13 \mu \mathrm{g} / \mathrm{kg}$, respectively. The recovery of analytes ranged from $61.28 \%$ to $116.20 \%$. The intra-day coefficient of variation (CV) was from 0.97 to $25.93 \%$ and the inter-day CV was 2.30-34.04\%. The method has been used for the determination of 49 residues/contaminants in bovine meat. Application of the method in routine analysis in bovine samples, revealed in limited samples the presences of enrofloxacin, oxytetracycline and sulfadiazine at the concentration of $35.22 \mu \mathrm{g} / \mathrm{kg}, 27.35 \mu \mathrm{g} / \mathrm{kg}$, and $36.20 \mu \mathrm{g} / \mathrm{kg}$, respectively.
\end{abstract}

Keywords: Residues, Contaminants, LC-MS/MS, Bovine meat, Validation

\section{Introduction}

Important source of nutrition for many people around the world is meat. The safety of meat is seriously challenged by all kinds of low molecular weight organic contaminants, such as residues of veterinary drugs, agro-chemical residues, mycotoxins, food additives and environmental contaminants, which arouse considerable attention from people over the world $[12,14]$. Liquid chromatography tandem mass spectrometry (LC-MS/MS) techniques provide a universal approach applicable to the widest number of veterinary drug residues and it is today become the technique for analysis of drug residues and contaminants in food stuffs. Until

\footnotetext{
*Correspondence: zhajrulai@fvm.ukim.edu.mk

${ }^{1}$ Faculty of Veterinary Medicine-Skopje, University "Ss. Cyril and Methodius", Lazar Pop-Trajkov 5/7, 1000 Skopje, Republic of North Macedonia

Full list of author information is available at the end of the article
}

10 years ago, many of the analytical methods mainly focused on the extraction and determination of a single class of analytes. In the monitoring programs the multiclass and multi-residue methods are increasingly being used because of their increased analytical scope and laboratory efficiency. The development of simultaneous multi-class drug residue determination is challenging task because concentrations of analyte are low in the tissues such as meat. Although the mass spectrometry is selective detection technique, the sample preparation step in analytical procedures is necessary because that reduces interference and matrix effect which occurs with the use of mass spectrometry, especially when using electrospray ionisation (ESI). Techniques such as immunoaffinity chromatography, liquid-liquid extraction, solid phase extraction (SPE) and matrix solid-phase dispersion (MSPD) are used for extraction and purification. Sometimes, for deconjugation 
or release of bound residues, in sample preparation the derivatization step can be incorporated $[3,13,19$, 23]. Muscle, such as other foods from animal origin, is a complex matrix. Thus, it is critical to use an efficient preparation method for sample extraction, clean-up, and concentration (when needed) before instrumental analysis. Extraction and clean-up are crucial steps in achieving the satisfactory recovery and purifying effect simultaneously for different classes of compounds from samples such as meat. However, the high concentration of proteins in meat complicates extraction and clean-up: cell compartmented enzymes (released during homogenization) can degrade some analytes during the extraction process. Proteins can also precipitate during clean-up (through solvent exchange) and cause irreversible adsorption of some analytes. Suitable sample preparation methods are therefore very critical. One such method is the QuEChERS (quick, easy, cheap, effective, rugged, and safe), originally developed for pesticide analysis [1], has also been applied to the determination of multi-class veterinary drugs in different food commodities [2, 17, 18, 20]. However, QuEChERS seems to be less suited for recovering polar veterinary drugs such as penicillins, tetracyclines and quinolones. Therefore, there is still a great need for simple and rapid multi-residue analytical methods with appropriate sample preparation techniques, for simultaneously determining veterinary drug residues, pesticides and mycotoxins in foods. Information on levels of these hazards in commonly consumed matrices is not frequently reported hence the need for development of appropriate methods to support generation of data to fill the gap. Only few methods, combining multi-detection and multi-class residues (veterinary) in a quantitative method for bovine muscle have been reported in literature. Biselli et al. [3] developed an LC-MS/MS method with a simple extraction procedure based on liquid extraction with ethylenediamine tetraacetic acidsuccinate buffer and acetronitrile $(\mathrm{MeCN})$. This method is suitable for determination of 84 veterinary drug residues in chicken muscle, including the following classes: benzimidazoles, quinolones, nitromidazoles, $\beta$-lactams, macrolides, triphenylmethane dyes, sulphonamides and tetracyclines. For determination of veterinary drug residues and contaminants in muscle or infant formula, in recent years, the low-temperature clean-up method has been widely developed $[21,23]$. Most of the lipid components can be successfully separated from extracts with the low-temperature clean-up method. The present paper describes a sensitive and reliable LC-MS/ MS including simple and generic method for the analysis of 49 veterinary drug residues and other contaminants in bovine meat.

\section{Materials and methods \\ Reference standards}

Amoxicillin (99.6\%), ampicillin (99.8\%), benzylpenicillin (99.3\%), cloxacillin (98.7\%), oxacillin (98.4\%), clenbuterol $\mathrm{HCl}(99.1 \%)$, isoxsuprine $\mathrm{HCl}(100 \%)$, salbutamol (99.4\%), zilpaterol $\mathrm{HCl}(96.0 \%)$, ractopamine $\mathrm{HCl}(95.5 \%)$, terbutaline hemisulfate salt (100.0\%), taleranol (99.5\%), 19 nortestosterone (99.8\%), clostebol (99.1\%), boldenone (99.1\%), methyltestosterone (99.5\%), testosterone (100.0\%), carbofuran (99.9\%), carbaryl (99.9\%), parathion (99.7\%), malathion (99.2\%), diazinon (98.3\%), dimethoate (99.8\%), atrazine (99.5\%), cypermethrin $(98.4 \%)$, permethrin (98.1\%), deltamethrin (99.9\%), coumaphos (99.7\%), dicholphos (99.8\%), chlorpyrifos (99.8\%), fenvalerate (99.4\%) were purchased Sigma-Aldrich (St. Louis, MO, USA). Brombuterol (98.0\%), mabuterol $\mathrm{HCl}$ (98.0\%), cimbuterol (98.0\%), clenpenterol $\mathrm{HCl}(98.0 \%)$ were obtained from Witega (Berlin, Germany). Zeranol (99.9\%), stanozolol (99.8\%), ceftioflur (98.0\%), cephalexin (96.6\%), oxytetracycline (96.5\%), enrofloxacin (99.74\%), ciprofloxacin (98.0\%), sulfadimidine (99.6\%), sulfamethoxazole (99.7\%), sulfadiazine (99.8\%), sulfachloropiridazine (99.1\%) and sulfadimethoxine (99.7\%) were obtained from Dr. Ehrenstorfer $\mathrm{GmbH}$ (Augsburg, Germany); ochratoxin A ( $\geq$ 98.0\%) and zearalenon (99.0\%) were obtained from Trylogy Analytical Laboratory, Inc. (Washington, USA).

\section{Isotopic labelled internal standards}

Clenbuterol-d6 $\mathrm{HCl}$ (98.0\%), brombuterol-d9 $\mathrm{HCl}$ (98.0\%), mabuterol-d9 $\mathrm{HCl}$ (98.0\%), clenpenterol-d5 $\mathrm{HCl}$ (98.0\%), cimbuterol-d9 (98.0\%), were obtained from Witega (Berlin, Germany); isoxsuprine-d5 hemifumarate $(\geq 98.0 \%)$ and ractopamine- $\mathrm{d} 6 \mathrm{HCl}(\geq 98.0 \%)$ were obtained from the European Reference Laboratory (EURL) at RIKILT, The Netherlands, salbutamol (albuterol)-d9 ( $\geq 98.0 \%$ ) was obtained from Dr. Ehrenstorfer $\mathrm{GmbH}$ (Augsburg, Germany); zilpaterol-d7 $(\geq 98.0 \%)$ and $\beta$-zearalenol-d4 $(\geq 98.0 \%)$ were obtained from Toronto Research Chemicals Inc. (Toronto, Canada), while terbutaline-d9 acetate hemihydrate (99.3\%), flunixin-d3 (100.0\%) and penicillin G-d7 N-ethylpiperidinium (98.1\%) salt were obtained from Sigma-Aldrich (St. Louis, MO, USA).

\section{Preparation of standard solutions}

Individual stock standard solutions and internal standards were prepared in methanol $(\mathrm{MeOH})$ at concentration from 0.528 to $3.610 \mathrm{mg} / \mathrm{mL}$, while the concentration of ochratoxin A was $50 \mu \mathrm{g} / \mathrm{mL}$ and for zearalenone was $100 \mu \mathrm{g} / \mathrm{mL}$ after reconstruction in methanol. After preparation of individual stock standard solutions and internal standards, the standards were divided in groups in accordance with the Maximul Recommended Residue 
Levels (MRL) and Minimum Required Performance Limits (MRPL) values according to the acceptance criteria of European Commision [6], European Commision [7], European Commision [8], SANCO [16], EU pesticide database [10].

The initial mixed working standards with concentration at $10 \mu \mathrm{g} / \mathrm{mL}$ were prepared in $\mathrm{MeOH}$ and kept at $-18{ }^{\circ} \mathrm{C}$. The groups of standards were: clenbuterol, brombuterol, mabuterol in group 1; cimbuterol, clenpenterol, isoxsuprine, ractopamine in group 2; salbutamol, terbutaline, zilpaterol in group 3; testosterone, methyltestosterone, boldenone, zeranol, 19 nortestosterone, stanozolol, clostebol, taleranol in group 4; amoxicillin, ampicillin, benzylpenicillin, cloxacillin, carbaryl, parathion, dimethoate, atrazine, permethrin, dicholphos, zearalenon, ochratoxin a in group 5; enrofloxacin, ciprofloxacin, oxytetracycline, sulfadimidine, sulfamethoxazole, sulfadimethoxine, sulfadiazine, sulfachloropiridazine in group 6; carbofuran, chlorpyrifos in group 7; malathion, diazinon, coumaphos in group 8. Working standards for oxacillin, cephalexin, deltamethrin and fenvalerate were prepared at $10 \mu \mathrm{g} / \mathrm{mL}$ and ceftioflur and cypermethrin at $100 \mu \mathrm{g} / \mathrm{mL}$.

Mixed internal standard working solutions including brombuterol-d9 $\mathrm{HCl}$, cimbuterol-d9, clenbuterol-d6 $\mathrm{HCl}$, clenpenterol-d5 $\mathrm{HCl}$, isoxsuprine-d5 hemifumarate, mabuterol-d $9 \mathrm{HCl}$, ractopamine- $\mathrm{d} 6 \mathrm{HCl}$, salbutamol (albuterol)- $\mathrm{d} 9$, terbutaline- $\mathrm{d} 9$ acetate hemihydrate, zilpaterol-d7) at $100 \mathrm{ng} / \mathrm{mL}$ and ( $\beta$-zearalenol-d4, flunixin$\mathrm{d} 3$ and penicillin $\mathrm{g}-\mathrm{d} 7)$ at $10 \mu \mathrm{g} / \mathrm{mL}$ were prepared and kept at $-18^{\circ} \mathrm{C}$.

\section{Chemicals and reagents}

LC-MS/MS grade $\mathrm{MeCN}$, water and $\mathrm{MeOH}$, HPLC grade ethylacetate (EtOAc), dichloromethane, ammonium hydroxide, $\mathrm{n}$-hexane, acetic acid, ammonium acetate were obtainedfrom Carlo Erba Reagent S.A.S (Val de Reuil, France); LC-MS/MS grade formic acid was from Merck (Darmstad, Germany and Oasis HLB cartridge (500 mg/6 mL) from Waters (Milford, MA, USA).

\section{Sample preparation}

A grand total of 100 random samples of fresh bovine meat were collected from local markets of North Macedonia. The collected samples were transported directly to the laboratory at $4{ }^{\circ} \mathrm{C}$ and then subjected to the following examination. All collected samples were homogenized. After homogenization, all samples were mixed and prepared one sample which was used for validation purposes. This process was intended to acquire appropriate-representative control sample. The control sample was checked with the analytical method to avoid preexisting contamination with the analytes of the method's scope. In the next step, $10 \mathrm{~g}$ of meat sample was fortified with the analytes and the internal standards and let to stand for $20 \mathrm{~min}$. Then, $20 \mathrm{~mL}$ of extraction mixture (MeCN:EtOAc:acetic acid, 49.5:49.5:1, v/v/v) was added and shaken vigorously for $1 \mathrm{~min}$ on a vortex mixer. The mixture was then shaken for $30 \mathrm{~min}$ with an automated shaker and centrifuged at $8000 \mathrm{rpm}$ for $10 \mathrm{~min}$, at $0{ }^{\circ} \mathrm{C}$. The extraction step was repeated and the combination of the supernatants was transferred to a $50 \mathrm{~mL}$ tube and kept at $-80{ }^{\circ} \mathrm{C}$, for $20 \mathrm{~min}$. The solution was filtered through filter paper ( $>95 \% \alpha$-cellulose content, dia. $240 \mathrm{~mm}$ ) and evaporated to nearly dryness under stream of nitrogen in a water bath at $35^{\circ} \mathrm{C}$. The extract was dissolved in $10 \mathrm{~mL}$ of $\mathrm{MeOH}$ :water (10:90, v/v) and the solution shaken for $1 \mathrm{~min}$ on a vortex mixer followed by cleanup by solid phase extraction using Oasis HLB cartridges. The cartridge was activated and conditioned by passing through $5 \mathrm{~mL}$ of $\mathrm{MeOH}$ and $5 \mathrm{~mL}$ water before supernatant was loaded and cartridges washed with $5 \mathrm{~mL}$ of water, and then vacuum-dried for $10 \mathrm{~min}$. The residues were eluted into a test tube using $4 \mathrm{~mL}$ $\mathrm{MeOH}: \mathrm{MeCN}$ :ammonium hydroxide (47.5:47.5:5, v/v/v) followed by $4 \mathrm{~mL} \mathrm{MeOH}$ :dichloromethane (30:70, v/v). The samples were evaporated to dryness under stream of nitrogen at $35{ }^{\circ} \mathrm{C}$ and the residue reconstituted with $1 \mathrm{~mL}$ of MeCN:water (10:90, v/v). Defatting was attained by adding $3 \mathrm{~mL}$ of $\mathrm{n}$-hexane of the reconstituted residues in $1 \mathrm{~mL}$ on $\mathrm{MeCN}$ :water. The solution was shaken vigorously for $1 \mathrm{~min}$ on a vortex mixer. After that, the lower layer was centrifuged at $10,000 \mathrm{rpm}$ for $3 \mathrm{~min}$ and the extract was filtered through a $0.45 \mu \mathrm{m}$ membrane filter into autosampler vials prior to LC-MS/MS analysis.

\section{LC-MS/MS analysis}

The analysis was performed with a Waters (Milford, MA, USA) quadrupole LC-MS/MS equipped with a binary pump, vacuum degasser, thermostated autosampler and thermostated column manager. MassLynx software (Waters, Milford, MA, USA) version 4.1 was used for instrument control, data acquisition and calculation of results. Chromatographic separation was carried out using an Kinetex C18 column $(50 \times 2.1 \mathrm{~mm}, 2.6 \mu \mathrm{m}$, Phenomenex, Torrance, CA, USA).

A gradient mobile phase was used where mobile phase A consisted of $0.1 \%$ formic acid solution in water containing $5 \mathrm{mM}$ ammonium acetate and mobile phase $\mathrm{B}$ consisted of $0.1 \%$ formic acid solution in $\mathrm{MeCN}$. The elution program was as follows: $0-1 \mathrm{~min}, 95-80 \% \mathrm{~A} ; 1-4 \mathrm{~min}$, 80-60\% A; 4-8 min, 60-95\% A; 8-12 min, 95\% A while the flow rate was $0.2 \mathrm{~mL} / \mathrm{min}$. The column temperature was $40^{\circ} \mathrm{C}$ and injection volume was $10 \mu \mathrm{L}$. 
The MS/MS acquisition was carried out using electrospray ionization positive and negative mode (ESI + and ESI -). The main MS conditions were optimized and finally set as follows: capillary voltage of $3.0 \mathrm{kV}$; source temperature of $150{ }^{\circ} \mathrm{C}$; desolvation temperature of $400{ }^{\circ} \mathrm{C}$; cone gas at $100 \mathrm{~L} / \mathrm{h}$; and desolvation gas at 300 $\mathrm{L} / \mathrm{h}$. Three multiple reaction monitoring (MRM) transitions of banned analytes were chosen, while for analytes with permitted limits, 2 MRM transitions were chosen. Analysis of internal standards involved one MRM transition. The optimized MRM conditions for each analyte are given in Table 1.

\section{Method validation}

The analytical method was validated according to the European Commission decision 2002/657 and validation approach of the SANTE/12682/2019 guidelines [9]. For the method validation were elaluated various parameters: linearity, accuracy, precision, limit of detection (LOD), limit of quantification (LOQ), Decision limit (CC $\alpha)$ and Detection capability $(C C \beta)$. The linearity of the method was evaluatedon the basis of matrix-match calibration. To assess the trueness of the analytical method, recovery study was conducted, considering also that certified reference materials (CRMs) not available.

\section{Results and discussion}

\section{Optimization of the MS/MS parameters}

Multi-class and multi-residue LC-MS/MS analytical method, was developed for simultaneous analysis in the single run in (electrospray ionisation) ESI + and ESI -. For both ionization modes, the best sensitivity for all drugs was determined to provide the highest signals for quantification and confirmation. In positive ion mode were detected $93.55 \%$ of compounds, while in negative ionization mode were identified $6.45 \%$ of targeted compounds. The performance criteria for the analytical methods for detection of residues are prescribed in Commission Decision 2002/657/EC, therefore, this document is crucial for consultation from the laboratories for analyses of residues. According to this document, for the confirmation of banned substances (Group A) a minimum of four identification points are required, while for the confirmation of permitted substances (Group B) the minimum number of identification points is set to three. To achive maximum response for all compounds the individual standard solution with concentration from $1 \mu \mathrm{g} / \mathrm{mL}$, was injected directly in the MS/MS detector. The MS/MS optimization parameters are summarized Table 1 . The daughter ion with the highest intensity was used for quantification of the compounds. The quantification ion for all compounds is indicated with bold character in Table 1. The best dwell time used was between 0.01 and $0.025 \mathrm{~s}$ whereby good peak shapes and suitable signal to noise ratio $(\mathrm{S} / \mathrm{N})$ were attained.

\section{LC optimization}

In order to achieve high sensitivity, good ionization, and sufficient separation with minimum interference from matrix, mobile phase and gradient are very important parameters in LC method. Different mobile phases were tested to provide the best chromatographic results, consisting of $\mathrm{MeOH}$ with $0.1 \%$ formic acid or $\mathrm{MeCN}$ with $0.1 \%$ formic acid, $\mathrm{MeOH}$ and $\mathrm{MeCN}$ (1:1) with $0.1 \%$ formic acid and water acified with formic acid $(0.01 \%$ and $0.1 \%, \mathrm{v} / \mathrm{v}$ ) and $5 \mathrm{mmol} / \mathrm{L}$ ammonium acetate. According to Ren et al. [15], Xie et al. [21] and Zhan et al. [23] formic acid or acetic acid could improve the ionization of analytes under ESI + mode, and ammonium formate or ammonium acetate could change the $\mathrm{pH}$ of mobile phase to improve the ionization of target compounds under ESI- mode.

In this study, the best sensitivity with better chromatographic separation and peak shape was attained using water with $0.1 \%$ formic acid and $5 \mathrm{mmol} / \mathrm{L}$ ammonium acetate as aqueous phase and $\mathrm{MeCN}$ with $0.1 \%$ formic acid as organic phase. Also, the peak shape and tailing were improved with addition of $5 \mathrm{mM}$ ammonium acetate. Due to the differences in physiochemical characteristics of target compounds, a gradient program described in section LC-MS/MS analysis was applied in order to elute 49 compounds within $12 \mathrm{~min}$.

\section{Optimization of the extraction procedure}

The optimization of extraction methods for multi-class veterinary drugs and contaminants is challenging in part because of differences in structures and physicochemical properties; potential interfering matrices and fats/lipid and proteins in foods such as meat. Thus, the sample preparation protocol that includes simultaneous extraction of all components is an important part of a multiresidue and multi-class analysis.

Better recoveries were obtained for most of the compounds when a mixture of $\mathrm{MeCN}$ and EtOAc acidified with $1 \%$ acetic acid was used. The effect of sample amount (5 $\mathrm{g}$ and $10 \mathrm{~g}$ ) and extraction steps on recovery was investigated (The results are given in Additional file 1: Table S1). The result showed that when $10 \mathrm{~g}$ of sample was extracted with twice $20 \mathrm{~mL}$ of MeCN:EtOAc:acetic acid $(49.5: 49.5: 1, \mathrm{v} / \mathrm{v} / \mathrm{v})$, the recovery of 61.3 to $116 \%$ was 
Table 1 MRM parameters for 49 compounds and 13 internal standards

\begin{tabular}{|c|c|c|c|c|c|}
\hline Standard & $\begin{array}{l}\text { lonisation mode } \\
\text { (ESI) }\end{array}$ & Precursor ion (m/z) & Product ion $(\mathrm{m} / \mathrm{z})$ & Collision energy (V) & $\begin{array}{l}\text { Cone } \\
\text { voltage } \\
\text { (V) }\end{array}$ \\
\hline Clenbuterol & + & 276.97 & $\begin{array}{l}\mathbf{2 0 2 . 9 5} \\
131.87 \\
167.77\end{array}$ & $\begin{array}{l}16 \\
30 \\
30\end{array}$ & 22 \\
\hline Brombuterol & + & 366.90 & $\begin{array}{l}\mathbf{2 9 2 . 8 4} \\
211.42 \\
57.00\end{array}$ & $\begin{array}{l}20 \\
34 \\
38\end{array}$ & 26 \\
\hline Mabuterol & + & 310.95 & $\begin{array}{l}\mathbf{2 3 6 . 9 9} \\
216.96 \\
57.00\end{array}$ & $\begin{array}{l}18 \\
26 \\
30\end{array}$ & 24 \\
\hline Clenpenterol & + & 291.00 & $\begin{array}{l}\mathbf{2 0 2 . 9 2} \\
131.89 \\
167.79\end{array}$ & $\begin{array}{l}16 \\
30 \\
28\end{array}$ & 28 \\
\hline Isoxsuprin & + & 302.04 & $\begin{array}{l}\mathbf{1 6 4 . 0 1} \\
106.96 \\
120.95\end{array}$ & $\begin{array}{l}30 \\
16 \\
28\end{array}$ & 26 \\
\hline Cimbuterol & + & 234.03 & $\begin{array}{l}\mathbf{1 5 9 . 9 8} \\
142.94 \\
57.00\end{array}$ & $\begin{array}{l}16 \\
28 \\
26\end{array}$ & 22 \\
\hline Ractopamine & + & 302.04 & $\begin{array}{l}106.96 \\
\mathbf{1 6 4 . 0 1} \\
120.95\end{array}$ & $\begin{array}{l}28 \\
16 \\
24\end{array}$ & 24 \\
\hline Salbutamol & + & 240.03 & $\begin{array}{l}\mathbf{1 4 7 . 9 6} \\
165.98 \\
56.94\end{array}$ & $\begin{array}{l}20 \\
14 \\
24\end{array}$ & 22 \\
\hline Zilpaterol & + & 262.03 & $\begin{array}{l}185.01 \\
\mathbf{2 0 2 . 0 5} \\
156.98\end{array}$ & $\begin{array}{l}24 \\
22 \\
32\end{array}$ & 22 \\
\hline Terbutalin & + & 226.00 & $\begin{array}{l}\mathbf{1 5 2 . 0 0} \\
106.97 \\
170.00\end{array}$ & $\begin{array}{l}14 \\
30 \\
16\end{array}$ & 26 \\
\hline Testosterone & + & 289.16 & $\begin{array}{l}108.99 \\
96.95 \\
289.18\end{array}$ & $\begin{array}{l}24 \\
28 \\
28\end{array}$ & 36 \\
\hline Methyltestosterone & + & 303.22 & $\begin{array}{l}\mathbf{9 6 . 9 6} \\
109.0 \\
178.18\end{array}$ & $\begin{array}{l}28 \\
24 \\
24\end{array}$ & 36 \\
\hline Boldenone & + & 287.16 & $\begin{array}{l}\mathbf{1 2 1 . 0 3} \\
135.02 \\
171.20\end{array}$ & $\begin{array}{l}24 \\
16 \\
20\end{array}$ & 34 \\
\hline Zeranol & - & 321.03 & $\begin{array}{l}\mathbf{9 0 . 8 7} \\
40.90 \\
259.2\end{array}$ & $\begin{array}{l}40 \\
40 \\
36\end{array}$ & 74 \\
\hline Taleranol & - & 321.03 & $\begin{array}{l}\mathbf{9 0 . 8 7} \\
40.90 \\
259.2\end{array}$ & $\begin{array}{l}34 \\
40 \\
42\end{array}$ & 74 \\
\hline 19 Nortestosterone & + & 275.14 & $\begin{array}{l}109.0 \\
80.56 \\
93.18\end{array}$ & $\begin{array}{l}34 \\
26 \\
32\end{array}$ & 38 \\
\hline Stanozolol & + & 329.22 & $\begin{array}{l}\mathbf{8 0 . 9 5} \\
95.00 \\
121.0\end{array}$ & $\begin{array}{l}46 \\
46 \\
42\end{array}$ & 64 \\
\hline Clostebol & + & 323.16 & $\begin{array}{l}\mathbf{1 4 2 . 9 6} \\
130.98 \\
157.13\end{array}$ & $\begin{array}{l}26 \\
26 \\
22\end{array}$ & 40 \\
\hline Amoxicillin & + & 367.07 & $\begin{array}{l}159.96 \\
90.89\end{array}$ & $\begin{array}{l}16 \\
40\end{array}$ & 28 \\
\hline Ampicillin & + & 350.05 & $\begin{array}{l}\mathbf{1 5 9 . 9 4} \\
105.98\end{array}$ & $\begin{array}{l}20 \\
12\end{array}$ & 34 \\
\hline
\end{tabular}


Table 1 (continued)

\begin{tabular}{|c|c|c|c|c|c|}
\hline Standard & $\begin{array}{l}\text { lonisation mode } \\
\text { (ESI) }\end{array}$ & Precursor ion $(\mathrm{m} / \mathrm{z})$ & Product ion $(\mathrm{m} / \mathrm{z})$ & Collision energy (V) & $\begin{array}{l}\text { Cone } \\
\text { voltage } \\
\text { (V) }\end{array}$ \\
\hline Benzylpenicillin & + & 334.99 & $\begin{array}{l}90.96 \\
80.94\end{array}$ & $\begin{array}{l}42 \\
52\end{array}$ & 44 \\
\hline Cloxacillin & + & 435.94 & $\begin{array}{l}159.97 \\
276.96\end{array}$ & $\begin{array}{l}18 \\
14\end{array}$ & 26 \\
\hline Oxacillin & + & 402.05 & $\begin{array}{l}159.96 \\
243.03\end{array}$ & $\begin{array}{l}10 \\
12\end{array}$ & 24 \\
\hline Cefalexin & + & 347.99 & $\begin{array}{l}\mathbf{1 7 3 . 9 3} \\
157.89\end{array}$ & $\begin{array}{l}8 \\
16\end{array}$ & 30 \\
\hline Ceftiofur & + & 523.96 & $\begin{array}{l}241.00 \\
125.17\end{array}$ & $\begin{array}{l}16 \\
58\end{array}$ & 34 \\
\hline Enrofloxacin & + & 360.05 & $\begin{array}{l}245.09 \\
72.02\end{array}$ & $\begin{array}{l}30 \\
36\end{array}$ & 36 \\
\hline Ciprofloxacin & + & 332.01 & $\begin{array}{l}\mathbf{2 3 0 . 9 4} \\
245.05\end{array}$ & $\begin{array}{l}40 \\
28\end{array}$ & 38 \\
\hline Oxytetracycline & + & 460.97 & $\begin{array}{l}200.93 \\
\mathbf{4 2 6 . 0 2}\end{array}$ & $\begin{array}{l}38 \\
30\end{array}$ & 36 \\
\hline Sulfachloropyridazine & + & 284.90 & $\begin{array}{l}155.93 \\
91.93\end{array}$ & $\begin{array}{l}16 \\
34\end{array}$ & 28 \\
\hline Sulfadiazine & + & 250.97 & $\begin{array}{l}91.93 \\
155.93\end{array}$ & $\begin{array}{l}30 \\
14\end{array}$ & 28 \\
\hline Sulfadimetoxine & + & 310.97 & $\begin{array}{l}155.93 \\
\mathbf{9 1 . 9 3}\end{array}$ & $\begin{array}{l}20 \\
32\end{array}$ & 36 \\
\hline Sulfadimidine & + & 278.95 & $\begin{array}{l}185.93 \\
\mathbf{9 1 . 9 3}\end{array}$ & $\begin{array}{l}18 \\
36\end{array}$ & 34 \\
\hline Sulfamethoxazole & + & 253.91 & $\begin{array}{l}92.00 \\
155.94\end{array}$ & $\begin{array}{l}30 \\
16\end{array}$ & 28 \\
\hline Carbofuran & + & 222.1 & $\begin{array}{l}\mathbf{1 6 5 . 1} \\
123.0\end{array}$ & $\begin{array}{l}12 \\
22\end{array}$ & 32 \\
\hline Carbaryl & + & 202.0 & $\begin{array}{l}145.05 \\
\mathbf{1 2 7 . 0}\end{array}$ & $\begin{array}{l}10 \\
32\end{array}$ & 26 \\
\hline Parathion & + & 308.97 & $\begin{array}{l}148.89 \\
\mathbf{2 4 6 . 7 8}\end{array}$ & $\begin{array}{l}18 \\
14\end{array}$ & 48 \\
\hline Malathion & + & 331.1 & $\begin{array}{l}127.0 \\
98.93\end{array}$ & $\begin{array}{l}14 \\
26\end{array}$ & 30 \\
\hline Diazinon & + & 305.1 & $\begin{array}{l}\mathbf{1 6 9 . 0} \\
153.0\end{array}$ & $\begin{array}{l}22 \\
20\end{array}$ & 44 \\
\hline Dimethoate & + & 229.50 & $\begin{array}{l}\mathbf{1 9 8 . 8 3} \\
124.84\end{array}$ & $\begin{array}{l}10 \\
20\end{array}$ & 30 \\
\hline Atrazine & + & 216.0 & $\begin{array}{l}\mathbf{1 7 4 . 2 2} \\
104.14\end{array}$ & $\begin{array}{l}15 \\
30\end{array}$ & 32 \\
\hline Permethrin & + & 390.97 & $\begin{array}{l}355.02 \\
182.92\end{array}$ & $\begin{array}{l}6 \\
12\end{array}$ & 34 \\
\hline Cypermethrin & + & 433.0 & $\begin{array}{l}190.89 \\
90.92\end{array}$ & $\begin{array}{l}20 \\
12\end{array}$ & 28 \\
\hline Deltamethrin & + & 229.84 & $\begin{array}{l}\mathbf{1 9 8 . 8 3} \\
124.85\end{array}$ & $\begin{array}{l}30 \\
14\end{array}$ & 30 \\
\hline Coumaphos & + & 362.97 & $\begin{array}{l}306.86 \\
226.86\end{array}$ & $\begin{array}{l}26 \\
18\end{array}$ & 52 \\
\hline Dichlorophos & + & 221.0 & $\begin{array}{l}109.15 \\
\mathbf{1 2 7 . 1 4}\end{array}$ & $\begin{array}{l}18 \\
18\end{array}$ & 44 \\
\hline Chlorpyrifos & + & 351.78 & $\begin{array}{l}199.77 \\
\mathbf{2 9 6 . 8 2}\end{array}$ & $\begin{array}{l}18 \\
12\end{array}$ & 38 \\
\hline Fenvalerate & + & 419.97 & $\begin{array}{l}166.91 \\
\mathbf{1 2 4 . 8 8}\end{array}$ & $\begin{array}{l}14 \\
42\end{array}$ & 38 \\
\hline
\end{tabular}


Table 1 (continued)

\begin{tabular}{|c|c|c|c|c|c|}
\hline Standard & $\begin{array}{l}\text { lonisation mode } \\
\text { (ESI) }\end{array}$ & Precursor ion $(\mathrm{m} / \mathrm{z})$ & Product ion $(\mathrm{m} / \mathrm{z})$ & Collision energy (V) & $\begin{array}{l}\text { Cone } \\
\text { voltage } \\
\text { (V) }\end{array}$ \\
\hline Zearalenone & - & 316.97 & $\begin{array}{l}130.87 \\
\mathbf{1 7 4 . 9 1}\end{array}$ & $\begin{array}{l}30 \\
26\end{array}$ & 62 \\
\hline Ochratoxin A & + & 404.0 & $\begin{array}{l}358.1 \\
\mathbf{2 2 1 . 0}\end{array}$ & $\begin{array}{l}30 \\
10\end{array}$ & 46 \\
\hline Clenbuterol-d6 & + & 283.03 & 203.56 & 16 & 22 \\
\hline Brombuterol-d9 & + & 375.93 & 293.87 & 18 & 24 \\
\hline Mabuterol-d9 & + & 320.07 & 237.94 & 18 & 24 \\
\hline Clenpenterol-d5 & + & 296.00 & 203.10 & 16 & 24 \\
\hline Isoxsuprin-d5 hemifumarat & + & 308.15 & 168.05 & 16 & 26 \\
\hline Cimbuterol-d9 & + & 243.07 & 160.96 & 16 & 20 \\
\hline Ractopamin-d6 & + & 308.10 & 168.05 & 16 & 24 \\
\hline Salbutamol (Albuterol)-d9 & + & 249.08 & 148.59 & 20 & 24 \\
\hline Zilpaterol-d7 & + & 269.08 & 185.15 & 24 & 22 \\
\hline Terbutalin-d9 & + & 235.07 & 152.83 & 16 & 34 \\
\hline Flunixin-d3 & + & 300.03 & 263.98 & 36 & 28 \\
\hline Penicilin G-d7 & + & 455.16 & 114.02 & 10 & 32 \\
\hline$\beta$-zearalenol-d4 & - & 323.03 & 160.02 & 30 & 68 \\
\hline
\end{tabular}

achieved for a higher number of veterinary drugs, pesticides and mycotoxins (33 veterinary drugs, 14 pesticides, 2 mycotoxins). MeCN allows the precipitation of proteins and the removal of fats. However, a lot of lipid matrix also existed in extracts and to solve this problem removing the remaining fat, the low-temperature cleanup $-80{ }^{\circ} \mathrm{C}$ for $20 \mathrm{~min}$ was chosen. This method had been previously introduced to remove excess lipids in milk [21] and animal muscles [23] succesfully. The influence of clean-up on recoveries was also studied. In this case, three different clean up protocols were examined using DSC-MCAX (300 mg/6 mL) which contain octyl C8 and benzene sulfonic acid sorbents, Bond Elut C18 (500 mg/6 mL) which contain bonded C18 silica sorbent (octadecylsilane bonded to silica particles) and Oasis HLB (500 mg/6 mL) which contain $\mathrm{N}$-vinylpyrrolidone and divinylbenzene sorbent, with analysis of spiked samples at three concentration levels and six replicates per level. The results are presented in Table 2. Results showed that for some compounds, when in the examination were used DSC-MCAX and Bond Elut cartridges, the obtained recoveries are low. The worse result for recovery obtained with DSCMCAX cartridge was $51.45 \%$ for amoxicillin, while the lowest recovery obtained with Bond Elut cartridge was $50.12 \%$ for benzylpenicillin (Table 2). The best recoveries were obtained with Oasis HLB cartridge. For that reason, Oasis HLB cartridge with a hydrophilic-lipophilic balanced co-polymer of n-vinylpyrrolidone and divinylbenzenes, was chosen in the final method. [4], reported that the composition of these cartridges allows binding of acidic, basic, or neutral analytes. Prior to analysis with LC-MS/MS $3 \mathrm{~mL}$-hexane saturated with $\mathrm{MeCN}$ was added to remove the lipid material. The comparison with other methods for detection of common or similar residues and contaminants in meat are given in are given in Table 3. It should be noted that methods that can simultaneously detect veterinary drugs, pesticides and mycotoxins in meat are very rare.

\section{Validation of the method Linearity}

The linearity of the analytical method was determined at five differenct concentration levels for each compound. Therefore, matrix-matched calibration curve and the internal standard were utilized in the method for quantification to reduce the matrix effect. Although the method covered various different classes of veterinary pharmaceuticals, pesticides and mycotoxines the use of internal standards was only feasible for $\beta$-agonists, some antibiotics: amoxicilin, ampicilin, benzylpenicilin, oxacilin, cloxacilin, ciprofloxacin, enrofloxacinand for one mycotoxin- zearalenon (due to aspects of availability, cost, and convenience). The linear range and coefficient of corelation $\left(\mathrm{R}^{2}\right)$ for each compound are given in Table $4 . R^{2}$ values of most compounds was $\geq 0.990$, but in 13 compounds $R^{2}$ was below 0.99 . The results correspond with the results obtained from Kaufman et al. 2008 and can probably be explained by the enzymatic/chemical instability or the extreme polarity of the compounds. The 
Table 2 Recoveries per clean-up material

\begin{tabular}{|c|c|c|c|c|}
\hline \multirow[t]{2}{*}{ Analytes } & \multirow{2}{*}{$\begin{array}{l}\text { Added } \\
\text { concentration } \\
(\mu \mathrm{g} / \mathrm{kg})\end{array}$} & \multicolumn{3}{|l|}{ Recovery (\%) } \\
\hline & & DSC-MCAX & Bond Elut & Oasis HLB \\
\hline \multirow[t]{3}{*}{ Clenbuterol } & 0.05 & 88.64 & 91.34 & 104.00 \\
\hline & 0.25 & 82.15 & 77.36 & 105.20 \\
\hline & 0.50 & 92.44 & 84.12 & 102.20 \\
\hline \multirow[t]{3}{*}{ Brombuterol } & 0.05 & 71.34 & 80.64 & 75.64 \\
\hline & 0.25 & 69.75 & 85.22 & 92.01 \\
\hline & 0.50 & 81.32 & 81.34 & 95.34 \\
\hline \multirow[t]{3}{*}{ Mabuterol } & 0.05 & 80.52 & 82.15 & 76.00 \\
\hline & 0.25 & 87.24 & 74.22 & 92.00 \\
\hline & 0.50 & 83.81 & 91.30 & 95.40 \\
\hline \multirow[t]{3}{*}{ Clenpenterol } & 0.2 & 104.12 & 71.38 & 87.50 \\
\hline & 0.5 & 101.36 & 78.33 & 93.80 \\
\hline & 0.75 & 88.32 & 76.91 & 95.47 \\
\hline \multirow[t]{3}{*}{ Isoxsuprin } & 0.2 & 71.26 & 75.12 & 88.50 \\
\hline & 0.5 & 77.84 & 77.46 & 106.40 \\
\hline & 0.75 & 70.20 & 80.20 & 102.00 \\
\hline \multirow[t]{3}{*}{ Cimbuterol } & 0.2 & 81.46 & 77.86 & 95.47 \\
\hline & 0.5 & 82.15 & 81.52 & 99.40 \\
\hline & 0.75 & 91.46 & 80.16 & 94.53 \\
\hline \multirow[t]{3}{*}{ Ractopamine } & 0.2 & 74.12 & 81.36 & 96.50 \\
\hline & 0.5 & 84.56 & 115.32 & 104.40 \\
\hline & 0.75 & 80.26 & 88.12 & 102.93 \\
\hline \multirow[t]{3}{*}{ Salbutamol } & 0.5 & 101.46 & 71.36 & 108.00 \\
\hline & 0.75 & 111.36 & 77.22 & 94.93 \\
\hline & 1.0 & 100.06 & 74.15 & 86.60 \\
\hline \multirow[t]{3}{*}{ Zilpaterol } & 0.5 & 77.25 & 80.01 & 81.40 \\
\hline & 0.75 & 81.46 & 84.32 & 98.93 \\
\hline & 1.0 & 79.32 & 82.56 & 91.10 \\
\hline \multirow[t]{3}{*}{ Terbutaline } & 0.5 & 88.36 & 75.36 & 116.20 \\
\hline & 0.75 & 71.32 & 77.88 & 93.20 \\
\hline & 1.0 & 74.15 & 80.48 & 77.40 \\
\hline \multirow[t]{3}{*}{ Testosterone } & 1.0 & 89.32 & 71.22 & 71.50 \\
\hline & 3.0 & 88.36 & 78.36 & 71.66 \\
\hline & 5.0 & 81.26 & 77.58 & 71.60 \\
\hline \multirow[t]{3}{*}{ Boldenone } & 1.0 & 69.22 & 65.15 & 92.0 \\
\hline & 3.0 & 61.14 & 59.36 & 81.34 \\
\hline & 5.0 & 74.88 & 67.12 & 77.80 \\
\hline \multirow[t]{3}{*}{ Clostebol } & 1.0 & 81.36 & 75.14 & 104.0 \\
\hline & 3.0 & 88.32 & 82.88 & 109.34 \\
\hline & 5.0 & 85.14 & 80.61 & 100.80 \\
\hline \multirow{3}{*}{$\begin{array}{l}\text { Methyltestos- } \\
\text { terone }\end{array}$} & 1.0 & 85.26 & 74.15 & 80.0 \\
\hline & 3.0 & 81.34 & 71.33 & 72.67 \\
\hline & 5.0 & 95.16 & 85.14 & 90.20 \\
\hline \multirow[t]{3}{*}{ Stanozolol } & 1.0 & 64.36 & 62.11 & 93.0 \\
\hline & 3.0 & 67.36 & 65.66 & 91.33 \\
\hline & 5.0 & 60.22 & 71.33 & 89.20 \\
\hline \multirow{3}{*}{$\begin{array}{l}19 \text { nortestos- } \\
\text { terone }\end{array}$} & 1.0 & 79.46 & 81.36 & 105.0 \\
\hline & 3.0 & 74.16 & 72.15 & 113.67 \\
\hline & 5.0 & 88.12 & 77.48 & 104.20 \\
\hline
\end{tabular}

Table 2 (continued)

\begin{tabular}{|c|c|c|c|c|}
\hline \multirow[t]{2}{*}{ Analytes } & \multirow{2}{*}{$\begin{array}{l}\text { Added } \\
\text { concentration } \\
(\mu \mathrm{g} / \mathrm{kg})\end{array}$} & \multicolumn{3}{|c|}{ Recovery (\%) } \\
\hline & & DSC-MCAX & Bond Elut & Oasis HLB \\
\hline \multirow[t]{3}{*}{ Zeranol } & 1.0 & 81.46 & 70.22 & 75.0 \\
\hline & 3.0 & 77.25 & 71.36 & 82.00 \\
\hline & 5.0 & 92.18 & 81.35 & 71.80 \\
\hline \multirow[t]{3}{*}{ Taleranol } & 1.0 & 75.36 & 80.26 & 71.0 \\
\hline & 3.0 & 67.35 & 75.12 & 80.34 \\
\hline & 5.0 & 61.36 & 71.36 & 89.60 \\
\hline \multirow[t]{3}{*}{ Amoxicillin } & 25.0 & 57.46 & 54.36 & 61.89 \\
\hline & 50.0 & 52.11 & 59.64 & 61.28 \\
\hline & 75.0 & 51.46 & 55.12 & 79.50 \\
\hline \multirow[t]{3}{*}{ Oxacillin } & 150.0 & 81.46 & 77.56 & 106.22 \\
\hline & 300.0 & 74.13 & 71.32 & 92.83 \\
\hline & 450.0 & 77.85 & 71.88 & 94.08 \\
\hline \multirow[t]{3}{*}{ Cloxacallin } & 25.0 & 85.14 & 80.12 & 78.96 \\
\hline & 50.0 & 83.22 & 81.36 & 74.94 \\
\hline & 75.0 & 91.13 & 85.46 & 86.37 \\
\hline \multirow[t]{3}{*}{ Benzylpenicillin } & 25.0 & 66.14 & 50.12 & 85.44 \\
\hline & 50.0 & 59.23 & 55.14 & 102.56 \\
\hline & 75.0 & 56.77 & 51.36 & 95.39 \\
\hline \multirow[t]{3}{*}{ Ampicillin } & 25.0 & 65.17 & 70.64 & 75.64 \\
\hline & 50.0 & 61.22 & 74.32 & 82.90 \\
\hline & 75.0 & 69.22 & 75.12 & 96.00 \\
\hline \multirow[t]{3}{*}{ Ceftioflur } & 500.0 & 88.36 & 81.88 & 95.64 \\
\hline & 1000.0 & 81.23 & 82.56 & 92.32 \\
\hline & 1500.0 & 84.13 & 90.12 & 93.29 \\
\hline \multirow[t]{3}{*}{ Cephalexin } & 100.0 & 74.15 & 81.36 & 105.18 \\
\hline & 200.0 & 76.33 & 90.12 & 106.66 \\
\hline & 300.0 & 81.22 & 88.56 & 96.12 \\
\hline \multirow[t]{3}{*}{ Enrofloxacin } & 50.0 & 91.36 & 80.92 & 113.78 \\
\hline & 100.0 & 77.15 & 81.36 & 76.71 \\
\hline & 150.0 & 81.34 & 90.15 & 82.24 \\
\hline \multirow[t]{3}{*}{ Ciprofloxacin } & 50.0 & 108.12 & 95.16 & 81.13 \\
\hline & 100.0 & 113.56 & 103.12 & 83.35 \\
\hline & 150.0 & 104.18 & 97.22 & 92.81 \\
\hline \multirow[t]{3}{*}{ Oxytetracycline } & 50.0 & 81.36 & 77.36 & 79.08 \\
\hline & 100.0 & 97.32 & 77.18 & 77.25 \\
\hline & 150.0 & 75.14 & 81.36 & 85.56 \\
\hline \multirow{3}{*}{$\begin{array}{l}\text { Sulfachloropyri- } \\
\text { dazine }\end{array}$} & 50.0 & 78.65 & 88.22 & 107.27 \\
\hline & 100.0 & 77.23 & 90.12 & 82.92 \\
\hline & 150.0 & 81.56 & 78.15 & 95.12 \\
\hline \multirow[t]{3}{*}{ Sulfadiazine } & 50.0 & 88.15 & 101.32 & 96.98 \\
\hline & 100.0 & 96.15 & 108.17 & 74.94 \\
\hline & 150.0 & 92.11 & 97.36 & 90.61 \\
\hline \multirow{3}{*}{$\begin{array}{l}\text { Sulfadimetox- } \\
\text { ine }\end{array}$} & 50.0 & 104.32 & 85.12 & 74.72 \\
\hline & 100.0 & 92.11 & 91.36 & 85.66 \\
\hline & 150.0 & 93.14 & 90.12 & 94.37 \\
\hline
\end{tabular}


Table 2 (continued)

\begin{tabular}{|c|c|c|c|c|}
\hline \multirow[t]{2}{*}{ Analytes } & \multirow{2}{*}{$\begin{array}{l}\text { Added } \\
\text { concentration } \\
(\mu \mathrm{g} / \mathrm{kg})\end{array}$} & \multicolumn{3}{|c|}{ Recovery (\%) } \\
\hline & & DSC-MCAX & Bond Elut & Oasis HLB \\
\hline \multirow[t]{3}{*}{ Sulfadimidine } & 50.0 & 74.15 & 77.36 & 86.30 \\
\hline & 100.0 & 78.32 & 70.51 & 96.57 \\
\hline & 150.0 & 77.36 & 72.12 & 99.23 \\
\hline \multirow{3}{*}{$\begin{array}{l}\text { Sulfameth- } \\
\text { oxazol }\end{array}$} & 50.0 & 88.35 & 71.36 & 101.26 \\
\hline & 100.0 & 71.22 & 77.15 & 84.19 \\
\hline & 150.0 & 70.36 & 80.02 & 92.35 \\
\hline \multirow[t]{3}{*}{ Carbofuran } & 5.0 & 71.36 & 84.12 & 79.80 \\
\hline & 10.0 & 70.06 & 81.36 & 92.52 \\
\hline & 15.0 & 75.18 & 90.12 & 96.93 \\
\hline \multirow[t]{3}{*}{ Carbaryl } & 25.0 & 82.08 & 71.34 & 86.04 \\
\hline & 50.0 & 71.46 & 75.18 & 102.20 \\
\hline & 75.0 & 78.54 & 72.36 & 102.97 \\
\hline \multirow[t]{3}{*}{ Parathion } & 25.0 & 59.46 & 61.34 & 65.28 \\
\hline & 50.0 & 62.40 & 64.52 & 72.36 \\
\hline & 75.0 & 69.77 & 64.36 & 79.82 \\
\hline \multirow[t]{3}{*}{ Malathion } & 10.0 & 91.48 & 80.54 & 74.51 \\
\hline & 20.0 & 98.22 & 84.38 & 86.9 \\
\hline & 30.0 & 95.44 & 71.36 & 87.22 \\
\hline \multirow[t]{3}{*}{ Diazinon } & 10.0 & 84.56 & 81.56 & 92.14 \\
\hline & 20.0 & 78.13 & 77.46 & 99.65 \\
\hline & 30.0 & 79.18 & 76.13 & 93.70 \\
\hline \multirow[t]{3}{*}{ Dimethoate } & 25.0 & 67.46 & 70.18 & 65.84 \\
\hline & 50.0 & 60.02 & 71.36 & 71.56 \\
\hline & 75.0 & 62.15 & 72.15 & 79.80 \\
\hline \multirow[t]{3}{*}{ Atrazine } & 25.0 & 74.14 & 104.13 & 85.80 \\
\hline & 50.0 & 81.22 & 105.22 & 99.56 \\
\hline & 75.0 & 76.54 & 89.36 & 92.85 \\
\hline \multirow[t]{3}{*}{ Permethrin } & 25.0 & 92.01 & 91.14 & 104.52 \\
\hline & 50.0 & 88.46 & 88.54 & 102.96 \\
\hline & 75.0 & 88.18 & 89.13 & 104.17 \\
\hline \multirow[t]{3}{*}{ Cypermethrin } & 1000.0 & 78.69 & 70.64 & 99.65 \\
\hline & 2000.0 & 74.36 & 77.22 & 92.43 \\
\hline & 3000.0 & 81.08 & 72.15 & 97.39 \\
\hline \multirow[t]{3}{*}{ Deltamethrin } & 15.0 & 92.46 & 70.64 & 84.40 \\
\hline & 30.0 & 103.12 & 70.88 & 92.60 \\
\hline & 45.0 & 101.46 & 73.63 & 86.93 \\
\hline \multirow[t]{3}{*}{ Coumaphos } & 10.0 & 90.60 & 91.46 & 84.90 \\
\hline & 20.0 & 88.60 & 97.12 & 89.30 \\
\hline & 30.0 & 85.12 & 92.15 & 94.43 \\
\hline \multirow[t]{3}{*}{ Dichlorophos } & 25.0 & 101.35 & 88.36 & 95.36 \\
\hline & 50.0 & 95.17 & 81.22 & 95.15 \\
\hline & 75.0 & 97.36 & 82.15 & 97.49 \\
\hline \multirow[t]{3}{*}{ Chlorpyrifos } & 5.0 & 62.08 & 75.22 & 65.6 \\
\hline & 10.0 & 66.13 & 71.36 & 71.15 \\
\hline & 15.0 & 65.88 & 70.14 & 77.60 \\
\hline
\end{tabular}

Table 2 (continued)

\begin{tabular}{lclll}
\hline Analytes & $\begin{array}{l}\text { Added } \\
\text { concentration } \\
(\boldsymbol{\mu g} / \mathbf{k g})\end{array}$ & \multicolumn{3}{l}{ Recovery (\%) } \\
\cline { 3 - 5 } & 12.5 & 69.54 & 64.66 & 61.47 \\
\hline Fenvalerate & 25.0 & 61.32 & 59.14 & 81.4 \\
& 50.0 & 64.12 & 58.36 & 70.36 \\
Zearalenone & 25.0 & 74.36 & 78.34 & 81.84 \\
& 50.0 & 72.15 & 80.12 & 84.36 \\
& 75.0 & 72.88 & 88.14 & 93.07 \\
Ochratoxin A & 25.0 & 81.56 & 65.14 & 73.84 \\
& 50.0 & 83.12 & 64.22 & 67.15 \\
& 75.0 & 81.48 & 69.77 & 86.85 \\
\hline
\end{tabular}

coefficient of corelation revealed good linearity in the concentration range for each compound.

$L O D, L O Q, C C \alpha, C C \beta$ The decision limit $(C C \alpha)$ and the detection capability $(C C \beta)$ were calculated according to the 2002/657/EC requirements. The LODs were calculated from the lower concentrations of analyte i.e., the lower standards which were used for calibration curve $(n=6)$, and as mean value, plus 3.3 times of the calculated standard deviation and for LOQ this was plus 10 times of the calculated standard deviation. The $\mathrm{CC} \alpha$ values were in the range of $0.067-$ $2103.84 \mu \mathrm{g} / \mathrm{kg}$, while the range for $\mathrm{CC} \beta$ was from 0.083 to $2482.13 \mu \mathrm{g} / \mathrm{kg}$. The LOD values were in the range of $0.059-291.36 \mu \mathrm{g} / \mathrm{kg}$, while the range for LOQ was from 0.081 to $328.13 \mu \mathrm{g} / \mathrm{kg}$. The gained results showed good sensitivity because the values of the LOD, LOQ, CC $\alpha$ and $C C \beta$ were in accordance with the requirements of 2002/657/EC. The results are show in Table 4.

\section{Recovery and precision}

Recovery and intra-day precision were evaluated by preparing spiked samples at three different concentration levels using six replicates for each concentration level in one day, while inter-day precision were evaluated by analysis of spiked samples at the same concentration levels on three consecutive days, also in six replicates for each concentration level. The spiked concentration levels and results for recovery and precion are shown in Table 5. The chromatograms from spiked meat samples and the second level are shown in Fig. 1.

Statistical evaluation revealed that average recoveries were in the range of 61.28 (amoxicillin at $50 \mu \mathrm{g} / \mathrm{kg}$ ) to $120 \%$ (terbutaline at $0.5 \mu \mathrm{g} / \mathrm{kg}$ ) and CV for the intra-day precision were in the range of 0.97 (for permethrin at 


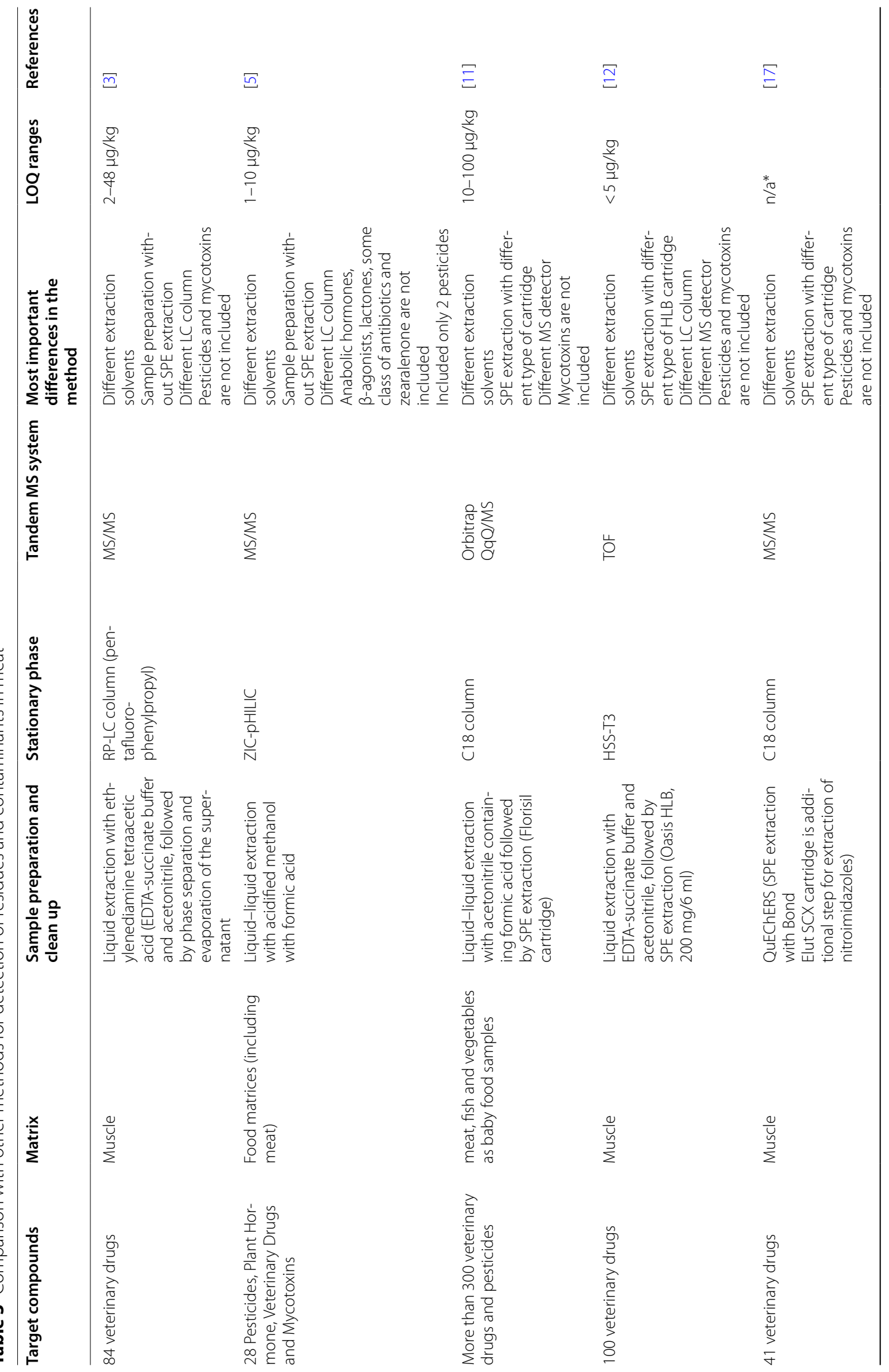




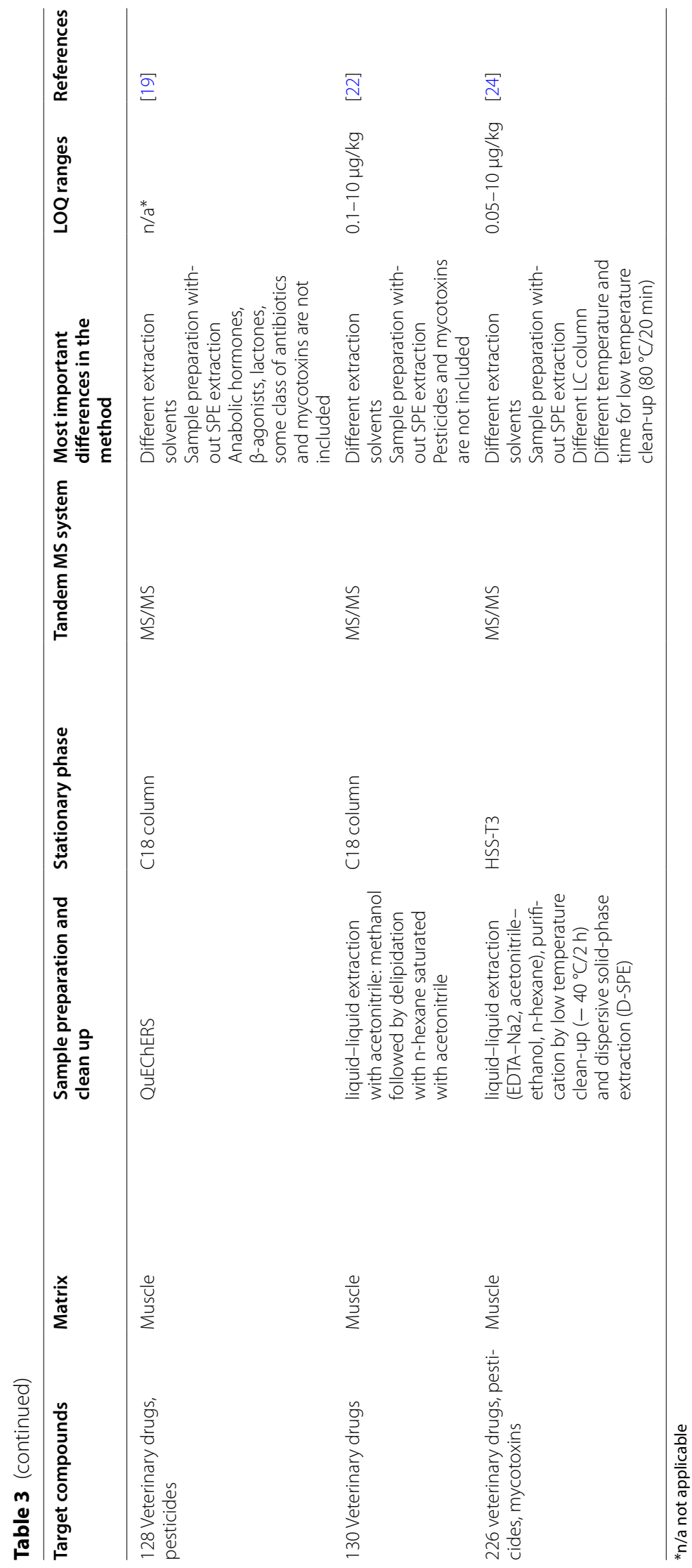


Table 4 Linear range, Coefficient of corelation, MRL/MRPL, CCa, CC $\beta$, LOD and LOQ

\begin{tabular}{|c|c|c|c|c|c|c|c|c|}
\hline Analytes & Linear range $(\mu \mathrm{g} / \mathrm{kg})$ & $\mathbf{R}^{2}$ & $\begin{array}{l}\mathrm{MRL}^{*} / \\
\mathrm{MRPL}^{* *} \\
(\mu \mathrm{g} / \mathrm{kg})\end{array}$ & $\mathrm{CCa}(\mu \mathrm{g} / \mathrm{kg})$ & $\mathrm{CC} \beta(\mu \mathrm{g} / \mathrm{kg})$ & $\operatorname{LOD}(\mu \mathrm{g} / \mathrm{kg})$ & LOQ $(\mu \mathrm{g} / \mathrm{kg})$ & Internal standard \\
\hline \multicolumn{9}{|l|}{$\beta$-agonists } \\
\hline Clenbuterol & $0.05-0.75$ & 0.990 & $0.1^{* *}$ & 0.068 & 0.083 & 0.059 & 0.081 & Clenbuterol-d6 \\
\hline Brombuterol & $0.05-0.75$ & 0.990 & $0.1^{* *}$ & 0.070 & 0.096 & 0.066 & 0.096 & Brombuterol-d9 \\
\hline Mabuterol & $0.05-0.75$ & 0.999 & $0.1^{* *}$ & 0.067 & 0.091 & 0.059 & 0.100 & Mabuterol-d9 \\
\hline Clenpenterol & $0.125-0.75$ & 0.995 & $0.5^{* *}$ & 0.298 & 0.421 & 0.290 & 0.410 & Clenpenterol-d5 \\
\hline Isoxsuprine & $0.125-0.75$ & 0.996 & $0.5^{* *}$ & 0.313 & 0.422 & 0.281 & 0.440 & $\begin{array}{l}\text { Isosuxprin-d5 } \\
\text { hemifumarat }\end{array}$ \\
\hline Cimbuterol & $0.125-0.75$ & 0.982 & $0.5^{* *}$ & 0.300 & 0.413 & 0.281 & 0.418 & Cimbuterl-d9 \\
\hline Ractopamine & $0.125-0.75$ & 0.981 & $1.0^{* *}$ & 0.192 & 0.264 & 0.218 & 0.307 & Ractopamin-d6 \\
\hline Salbutamol & $0.25-2.50$ & 0.983 & $5.0^{* *}$ & 1.083 & 1.398 & 0.546 & 0.732 & Salbutamol-d9 \\
\hline Zilpaterol & $0.25-2.50$ & 0.990 & $5.0^{* *}$ & 0.677 & 0.945 & 0.608 & 0.894 & Zilpaterol-d7 \\
\hline Terbutalin & $0.25-2.50$ & 0.982 & $10.0^{* *}$ & 0.840 & 1.111 & 0.616 & 0.897 & Terbutalin-d9 \\
\hline \multicolumn{9}{|l|}{ Anabolics } \\
\hline Testosteron & $1.0-50.0$ & 0.992 & - & 0.325 & 0.407 & 0.264 & 0.378 & \\
\hline Boldenon & $1.0-50.0$ & 0.998 & $1.0^{* *}$ & 0.428 & 0.522 & 0.308 & 0.419 & \\
\hline Clostebol & $1.0-50.0$ & 0.983 & - & 0.742 & 1.000 & 0.597 & 0.782 & \\
\hline $\begin{array}{l}\text { Methyltestoster- } \\
\text { one }\end{array}$ & $1.0-50.0$ & 0.9 & $1.0^{* *}$ & 0.695 & 0.855 & 0.504 & 0.690 & \\
\hline Stanozolol & $1.0-50.0$ & 0.981 & $1.0^{* *}$ & 0.548 & 0.745 & 0.498 & 0.672 & \\
\hline 19 nortestosterone & $1.0-50.0$ & 0.990 & $1.0^{* *}$ & 0.442 & 0.580 & 0.376 & 0.511 & \\
\hline \multicolumn{9}{|l|}{ Lactones } \\
\hline Zeranol & $1.0-50.0$ & 0.990 & $1.0^{* *}$ & 0.338 & 0.473 & 0.296 & 0.423 & \\
\hline Taleranol & $1.0-50.0$ & 0.999 & - & 0.715 & 0.900 & 0.578 & 0.792 & \\
\hline \multicolumn{9}{|l|}{$\beta$-lactams } \\
\hline Amoxicillin & $25.0-200.0$ & 0.991 & $50^{*}$ & 55.12 & 66.40 & 27.82 & 32.62 & Penicilin G-d7 \\
\hline Oxacillin & $50.0-500.0$ & 0.990 & $300^{*}$ & 312.33 & 359.22 & 46.22 & 49.53 & Penicilin G-d7 \\
\hline Cloxacilin & $25.0-200.0$ & 0.997 & - & 52.78 & 57.15 & 19.21 & 22.71 & Penicilin G-d7 \\
\hline Benzylpenicillin & $25.0-200.0$ & 0.983 & $50^{*}$ & 56.32 & 66.43 & 17.21 & 19.46 & Penicilin G-d7 \\
\hline Ampicillin & $25.0-200.0$ & 0.990 & $50^{*}$ & 54.12 & 59.31 & 22.31 & 24.36 & Penicilin G-d7 \\
\hline \multicolumn{9}{|l|}{ Cephalosporins } \\
\hline Ceftiofur & $300.0-3000.0$ & 0.991 & $1000^{*}$ & 1114.21 & 1272.8 & 291.36 & 328.13 & \\
\hline Cephalexin & $50.0-500.0$ & 0.990 & $200^{*}$ & 224.15 & 248.64 & 42.88 & 47.15 & \\
\hline \multicolumn{9}{|l|}{ Fluoroquinolones } \\
\hline Enrofloxacin & $25.0-200.0$ & 0.990 & $100^{*}$ & 105.22 & 113.94 & 24.84 & 28.07 & Flunixin d3 \\
\hline Ciprofloxacin & $25.0-200.0$ & 0.992 & $100^{*}$ & 108.17 & 132.72 & 25.32 & 29.91 & Flunixin d3 \\
\hline \multicolumn{9}{|l|}{ Tetracyclines } \\
\hline Oxytetracyclin & $25.0-200.0$ & 0.999 & $100^{*}$ & 111.45 & 116.74 & 20.78 & 23.48 & \\
\hline \multicolumn{9}{|l|}{ Sulfonamides } \\
\hline $\begin{array}{l}\text { Sulfachloropyri- } \\
\text { dazine }\end{array}$ & $25.0-200.0$ & 0.999 & $100^{*}$ & 112.15 & 128.91 & 26.90 & 31.31 & \\
\hline Sulfadiazine & $25.0-200.0$ & 0.983 & $100^{*}$ & 108.23 & 117.26 & 27.83 & 32.45 & \\
\hline Sulfadimethoxine & $25.0-200.0$ & 0.990 & $100^{*}$ & 111.14 & 129.22 & 27.11 & 30.76 & \\
\hline Sulfadimidine & $25.0-200.0$ & 0.990 & $100^{*}$ & 104.56 & 109.21 & 26.86 & 30.69 & \\
\hline Sulfamethoxazol & $25.0-200.0$ & 0.998 & $100^{*}$ & 122.18 & 134.95 & 26.69 & 29.89 & \\
\hline \multicolumn{9}{|l|}{ OP pesticides } \\
\hline Carbofuran & $1.0-50.0$ & 0.991 & $10^{*}$ & 12.26 & 13.81 & 1.08 & 2.12 & \\
\hline Carbaryl & $25.0-200.0$ & 0.990 & $50^{*}$ & 54.18 & 57.33 & 26.06 & 29.15 & \\
\hline Parathion & $25.0-200.0$ & 0.981 & $50^{*}$ & 59.12 & 62.78 & 21.36 & 24.35 & \\
\hline Malathion & $1.0-50.0$ & 0.990 & $20^{*}$ & 23.13 & 27.37 & 1.54 & 3.21 & \\
\hline
\end{tabular}


Table 4 (continued)

\begin{tabular}{|c|c|c|c|c|c|c|c|c|}
\hline Analytes & Linear range $(\mu \mathrm{g} / \mathrm{kg})$ & $R^{2}$ & $\begin{array}{l}\text { MRL*/ } \\
M R P L^{* *} \\
(\mu g / k g)\end{array}$ & $\mathrm{CCa}(\mu \mathrm{g} / \mathrm{kg})$ & $\operatorname{CC} \beta(\mu \mathrm{g} / \mathrm{kg})$ & $\operatorname{LOD}(\mu \mathrm{g} / \mathrm{kg})$ & LOQ $(\mu \mathrm{g} / \mathrm{kg})$ & Internal standard \\
\hline Diazinon & $1.0-50.0$ & 0.982 & $20^{*}$ & 25.47 & 31.19 & 1.48 & 2.54 & \\
\hline Dimethoate & $25.0-200.0$ & 0.990 & - & 55.17 & 63.63 & 16.25 & 19.23 & \\
\hline Atrazine & $25.0-200.0$ & 0.981 & - & 54.13 & 60.01 & 18.24 & 20.46 & \\
\hline Permethrin & $25.0-200.0$ & 0.999 & $50^{*}$ & 56.38 & 59.06 & 20.51 & 22.18 & \\
\hline Cypermethrin & $300.0-3000.0$ & 0.992 & $2000^{*}$ & 2103.84 & 2482.13 & 287.26 & 315.22 & \\
\hline Deltamethrin & $25.0-100.0$ & 0.994 & $30^{*}$ & 32.18 & 35.08 & 10.14 & 12.78 & \\
\hline Coumaphos & $1.0-50.0$ & 0.991 & $20^{*}$ & 22.15 & 25.49 & 2.48 & 3.76 & \\
\hline Dichlorophos & $25.0-200.0$ & 0.990 & - & 56.88 & 61.36 & 19.22 & 22.43 & \\
\hline Chlorpyrifos & $1.0-50.0$ & 0.981 & $10^{*}$ & 13.56 & 17.08 & 1.63 & 2.44 & \\
\hline Fenvalerate & $1.0-50.0$ & 0.983 & $25^{*}$ & 28.12 & 33.46 & 2.28 & 3.17 & \\
\hline \multicolumn{9}{|l|}{ Mycotoxins } \\
\hline Zearalenone & $25.0-200.0$ & 0.999 & - & 54.38 & 59.26 & 20.14 & 23.71 & $\beta$-zearalenol-d4 \\
\hline Ochratoxin A & $25.0-200.0$ & 0.990 & - & 57.44 & 62.18 & 19.21 & 22.14 & - \\
\hline
\end{tabular}

*MRL; **MRPL

$75 \mu \mathrm{g} / \mathrm{kg}$ ) to $25.93 \%$ (isoxsuprin at $0.2 \mu \mathrm{g} / \mathrm{kg}$ ). For interday precision the $\mathrm{CV}$ was from $2.27 \%$ (for cypermetrine at $3000 \mu \mathrm{g} / \mathrm{kg}$ ) to $34.04 \%$ (for ciprofloxacin at $50 \mu \mathrm{g} / \mathrm{kg}$ ). For all investigated compounds, the average recoveries and $\mathrm{CV}$ for precision was acceptable and in agreement with the criteria of Commission Decision 2002/657/EC and SANTE/12682/2019. To further demonstrate the suitability of the method the laboratory will be participate in proficiency tests in the near future.

\section{Real sample analysis}

A total of 87 import and local samples from bovine meat were analysed, in order to test the applicability of the established method for the routine analysis. After the sample analysis the trace residues of enrofloxacin, oxytetracycline and sulfadiazine at the concentration of $35.22 \mu \mathrm{g} / \mathrm{kg}, 27.35 \mu \mathrm{g} / \mathrm{kg}$, and $36.20 \mu \mathrm{g} / \mathrm{kg}$, respectively, were confirmed in three samples. The chromatograms from positive samples are given in Additional file 2: Figs. S1-S3.

\section{Conclusions}

A multi-class and multi-residue analytical methods including use of stable isotopes were developed for analysis of residues of veterinary drugs and other contaminants in bovine meat. The veterinary drugs included antimicrobials ( $\beta$-lactams, fluoroquinolones, cephalosporins, tetracyclines and sulphonamides), anabolic hormones, lactones, $\beta$-agonists and from pesticides and mycotoxins (zearalenone and ochratoxin A). Thirteen (13) stable isotopes were used as internal standards to cover wide range of analytes including veterinary drug residues, pesticides and mycotoxins. The developed method showed good performance characteristics of the method comply with EU recommendations. 
Table 5 Recovery and precision of the method

\begin{tabular}{|c|c|c|c|c|c|}
\hline Analytes & $\begin{array}{l}\text { Added concentration } \\
(\mu \mathrm{g} / \mathrm{kg})\end{array}$ & $\begin{array}{l}\text { Concentration in the samples } \\
(\mu \mathrm{g} / \mathrm{kg})\end{array}$ & Recovery (\%) & $\begin{array}{l}\text { Intra-day precision } \\
\mathrm{CV}_{\mathrm{r}}(\%)\end{array}$ & $\begin{array}{l}\text { Inter-day } \\
\text { precision } \mathrm{CV}_{\mathrm{R}} \\
(\%)\end{array}$ \\
\hline \multirow[t]{3}{*}{ Clenbuterol } & 0.05 & 0.052 & 104.00 & 5.54 & 16.49 \\
\hline & 0.25 & 0.263 & 105.20 & 4.27 & 7.02 \\
\hline & 0.50 & 0.511 & 102.20 & 14.05 & 7.74 \\
\hline \multirow[t]{3}{*}{ Brombuterol } & 0.05 & 0.038 & 75.64 & 13.22 & 17.74 \\
\hline & 0.25 & 0.220 & 92.01 & 4.17 & 12.50 \\
\hline & 0.50 & 0.477 & 95.34 & 9.96 & 11.81 \\
\hline \multirow[t]{3}{*}{ Mabuterol } & 0.05 & 0.038 & 76.00 & 4.73 & 10.27 \\
\hline & 0.25 & 0.230 & 92.00 & 3.37 & 5.87 \\
\hline & 0.50 & 0.477 & 95.40 & 8.23 & 10.37 \\
\hline \multirow[t]{3}{*}{ Clenpenterol } & 0.2 & 0.175 & 87.50 & 18.78 & 27.23 \\
\hline & 0.5 & 0.469 & 93.80 & 5.24 & 7.55 \\
\hline & 0.75 & 0.716 & 95.47 & 1.37 & 9.36 \\
\hline \multirow[t]{3}{*}{ Isoxsuprin } & 0.2 & 0.177 & 88.50 & 25.93 & 28.17 \\
\hline & 0.5 & 0.532 & 106.40 & 4.51 & 10.67 \\
\hline & 0.75 & 0.765 & 102.00 & 5.08 & 8.51 \\
\hline \multirow[t]{3}{*}{ Cimbuterol } & 0.2 & 0.177 & 95.47 & 9.89 & 15.99 \\
\hline & 0.5 & 0.497 & 99.40 & 10.27 & 14.58 \\
\hline & 0.75 & 0.709 & 94.53 & 11.33 & 17.63 \\
\hline \multirow[t]{3}{*}{ Ractopamine } & 0.2 & 0.193 & 96.50 & 6.33 & 11.32 \\
\hline & 0.5 & 0.522 & 104.40 & 10.13 & 11.63 \\
\hline & 0.75 & 0.772 & 102.93 & 6.57 & 9.69 \\
\hline \multirow[t]{3}{*}{ Salbutamol } & 0.5 & 0.540 & 108.00 & 11.66 & 14.72 \\
\hline & 0.75 & 0.712 & 94.93 & 22.13 & 24.83 \\
\hline & 1.0 & 0.836 & 86.60 & 15.03 & 20.48 \\
\hline \multirow[t]{3}{*}{ Zilpaterol } & 0.5 & 0.407 & 81.40 & 10.32 & 18.46 \\
\hline & 0.75 & 0.742 & 98.93 & 8.99 & 16.54 \\
\hline & 1.0 & 0.911 & 91.10 & 8.56 & 16.99 \\
\hline \multirow[t]{3}{*}{ Terbutaline } & 0.5 & 0.581 & 116.20 & 1.28 & 5.32 \\
\hline & 0.75 & 0.699 & 93.20 & 16.83 & 23.62 \\
\hline & 1.0 & 0.774 & 77.40 & 6.10 & 17.09 \\
\hline \multirow[t]{3}{*}{ Testosterone } & 1.0 & 0.715 & 71.50 & 8.39 & 13.65 \\
\hline & 3.0 & 2.15 & 71.66 & 8.84 & 14.61 \\
\hline & 5.0 & 3.58 & 71.60 & 6.70 & 9.26 \\
\hline \multirow[t]{3}{*}{ Boldenone } & 1.0 & 0.92 & 92.0 & 5.43 & 9.94 \\
\hline & 3.0 & 2.44 & 81.34 & 15.16 & 19.91 \\
\hline & 5.0 & 3.89 & 77.80 & 3.89 & 7.50 \\
\hline \multirow[t]{3}{*}{ Clostebol } & 1.0 & 1.04 & 104.0 & 12.50 & 15.6 \\
\hline & 3.0 & 3.28 & 109.34 & 8.54 & 18.84 \\
\hline & 5.0 & 5.04 & 100.80 & 4.17 & 6.70 \\
\hline \multirow[t]{3}{*}{ Methyltestosterone } & 1.0 & 0.80 & 80.0 & 5.00 & 8.34 \\
\hline & 3.0 & 2.18 & 72.67 & 9.64 & 14.44 \\
\hline & 5.0 & 4.51 & 90.20 & 15.75 & 18.51 \\
\hline \multirow[t]{3}{*}{ Stanozolol } & 1.0 & 0.93 & 93.0 & 5.38 & 9.39 \\
\hline & 3.0 & 2.74 & 91.33 & 3.29 & 7.02 \\
\hline & 5.0 & 4.46 & 89.20 & 11.44 & 15.09 \\
\hline \multirow[t]{3}{*}{19 nortestosterone } & 1.0 & 1.05 & 105.0 & 8.57 & 13.31 \\
\hline & 3.0 & 3.41 & 113.67 & 6.16 & 10.66 \\
\hline & 5.0 & 5.21 & 104.20 & 9.98 & 19.42 \\
\hline \multirow[t]{3}{*}{ Zeranol } & 1.0 & 0.75 & 75.0 & 9.34 & 21.49 \\
\hline & 3.0 & 2.46 & 82.00 & 3.66 & 5.78 \\
\hline & 5.0 & 3.59 & 71.80 & 11.42 & 14.09 \\
\hline
\end{tabular}


Hajrulai-Musliu et al. BMC Chemistry 2022, 15(1):62

Page 15 of 21

Table 5 (continued)

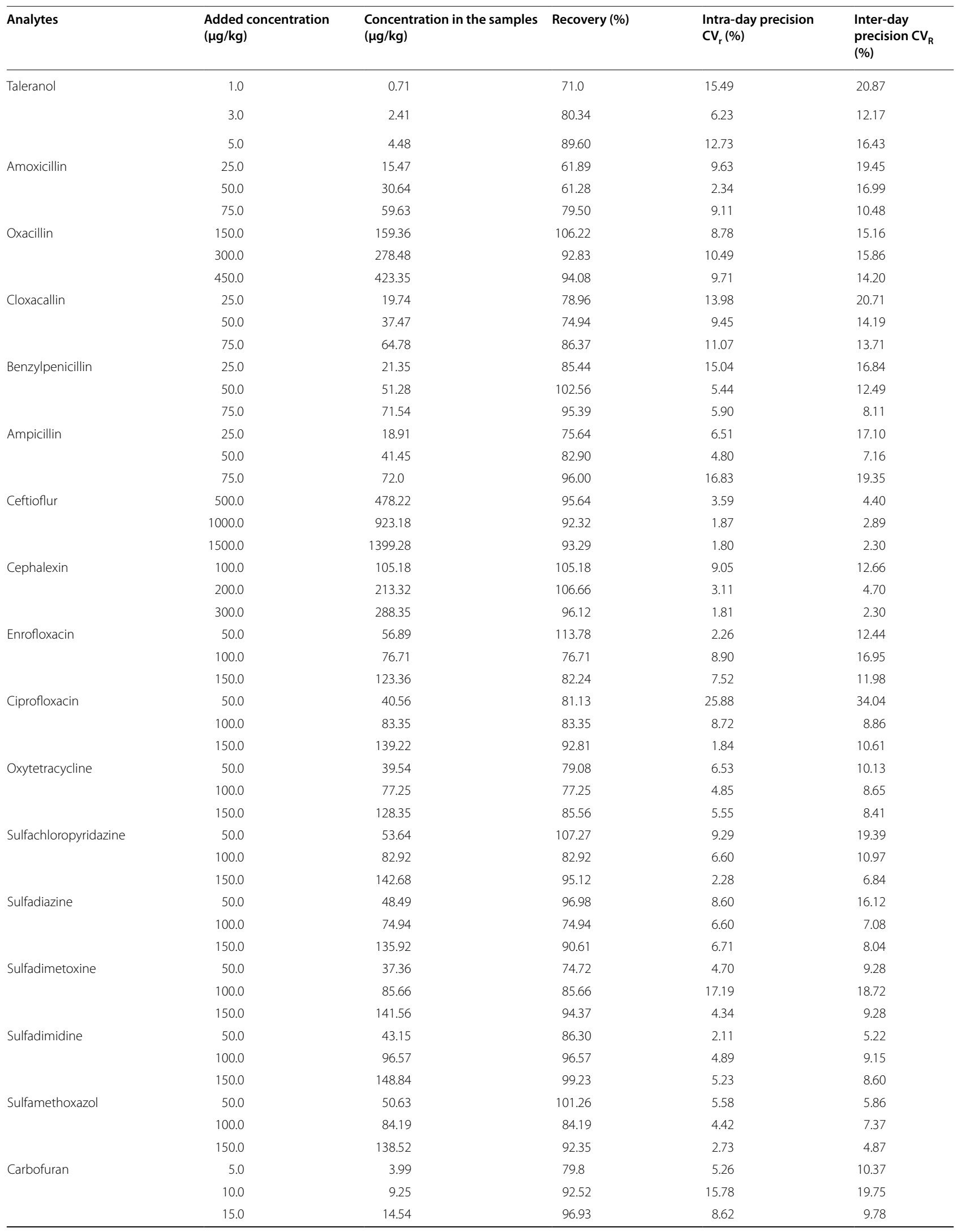


Hajrulai-Musliu et al. BMC Chemistry 2022, 15(1):62

Page 16 of 21

Table 5 (continued)

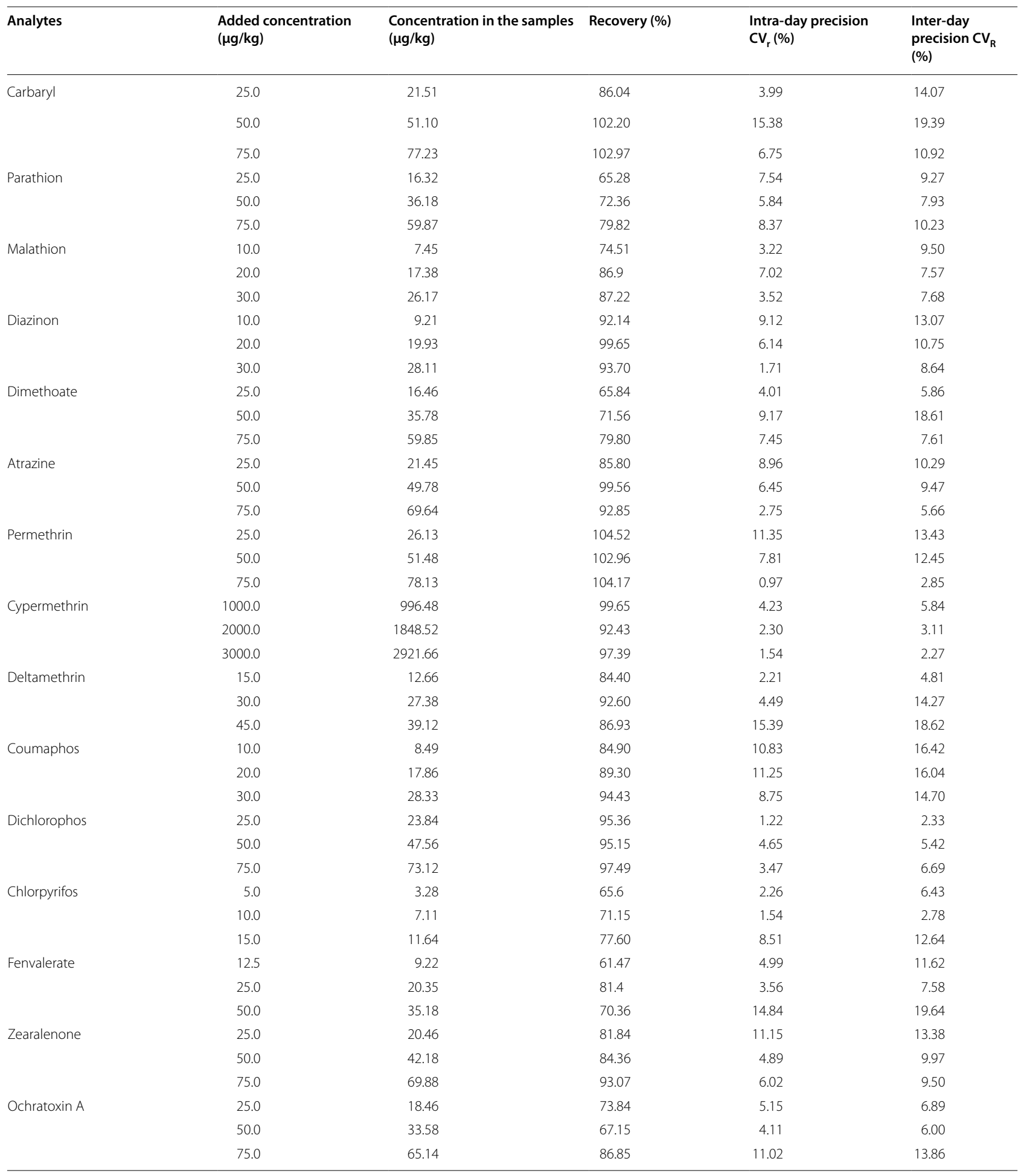




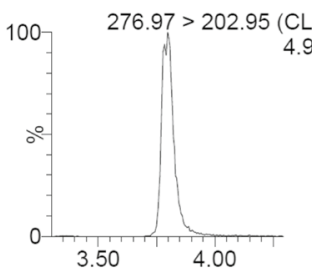

Clenbuterol (CLEN)
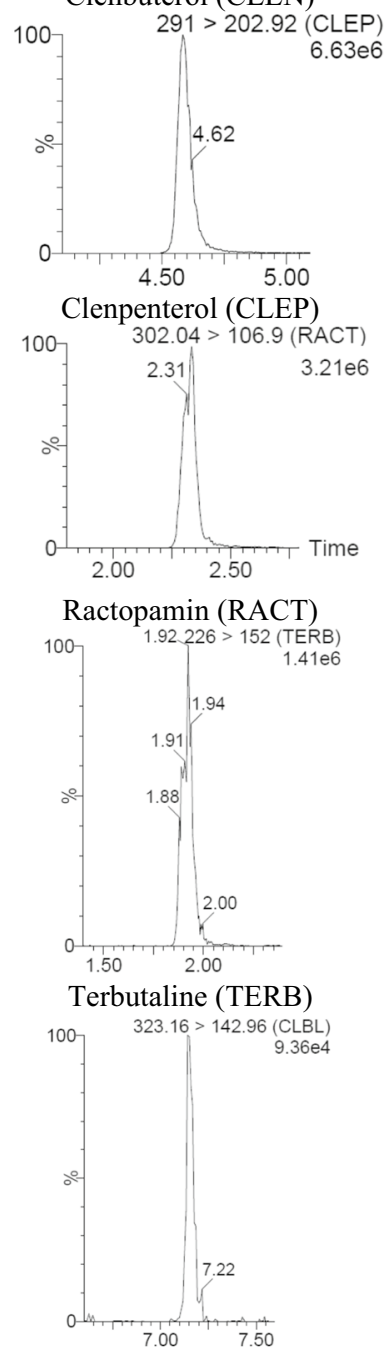

Clostebol (CLBL)

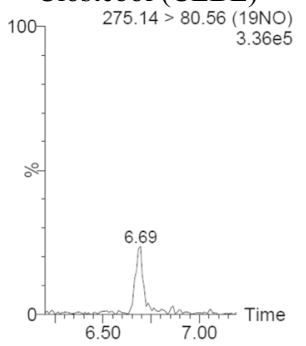

19 Nortestosteron (19NO)

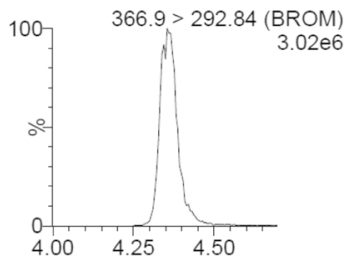

Brombuterol (BROM)
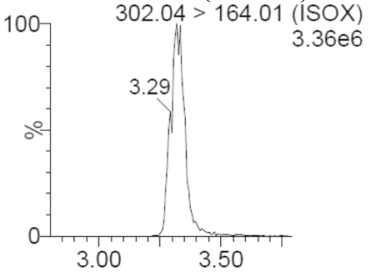

Isoxsuprin (ISOX)

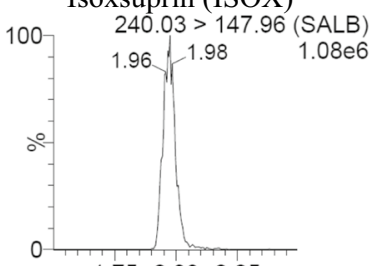

$\begin{array}{lll}1.75 & 2.002 .25\end{array}$
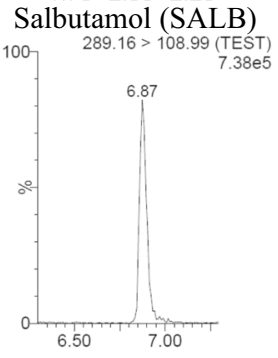

Testosteron (TEST)

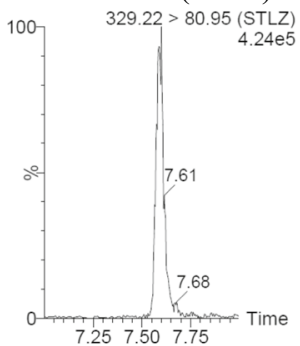

Stanozolol (STLZ)

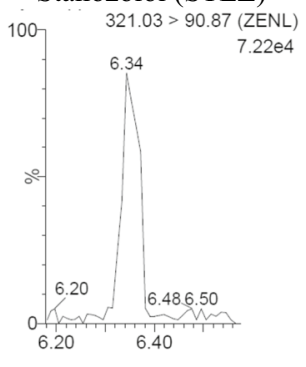

Zeranol (ZENL)

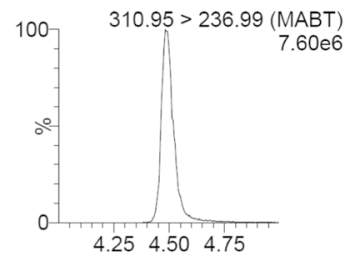

Mabuterol (MABT)

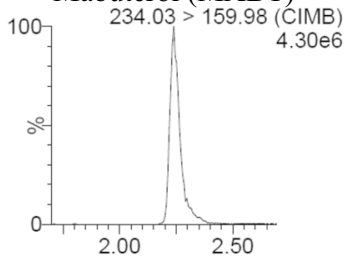

Cimbuterol (CIMB)

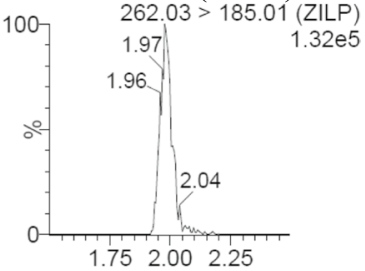

Zilpaterol (ZILP)

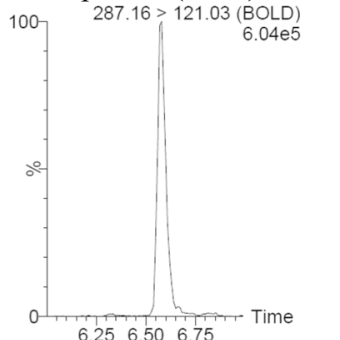

Boldenon (BOLD)

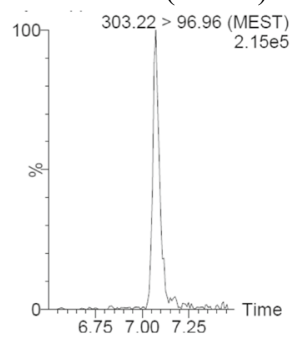

Methyltestosteron (MEST)

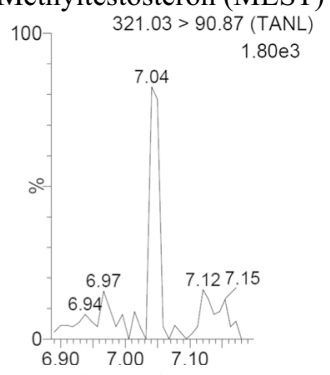

Taleranol (TANL)

Fig. 1 Chromatograms from spiked meat samples at the second concentration level (the second level for all analytes are given in Table 4) 


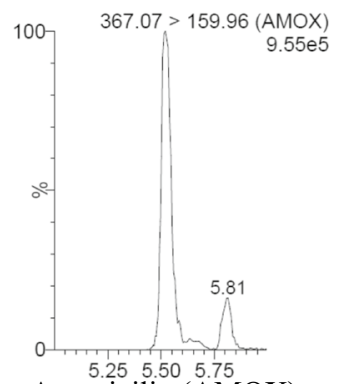

Amoxicilin (AMOX)

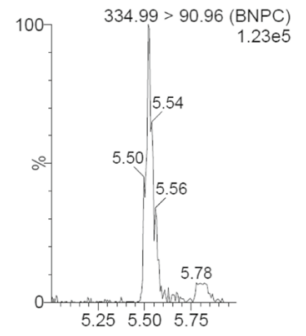

Benzylpenicilin (BNPC)

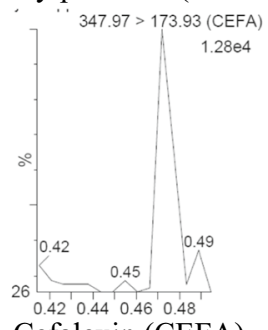

Cefalexin (CEFA)

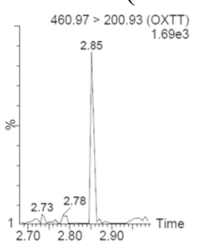

Oxytetracyclin (OXTT)

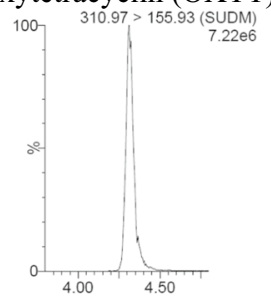

Sulfametoxin (SUDM)

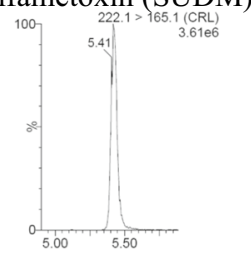

Carbofuran (CRL)

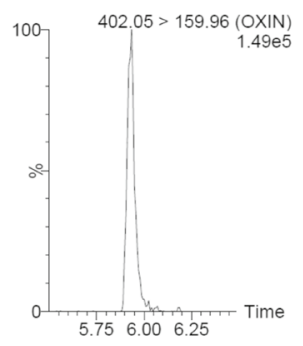

Oxacilin (OXIN)

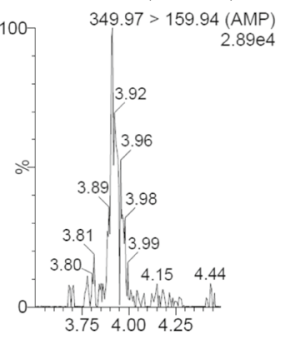

Ampicilin (AMP)
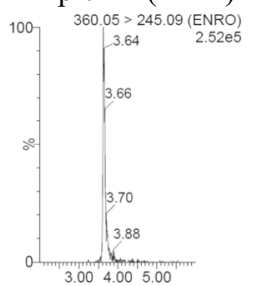

Enrofloxacin (ENRO)

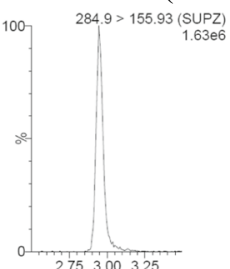

Sulfachloropyridazin (SUPZ)

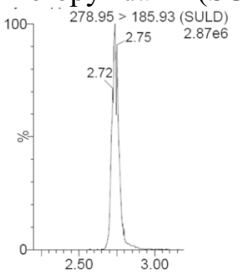

Sulfadimidin (SULD)

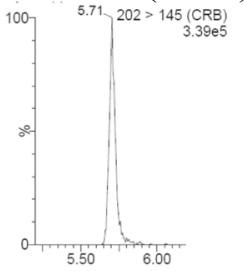

Carbaryl (CRB)

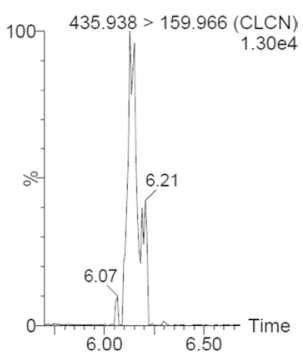

Cloxacilin (CLCN)

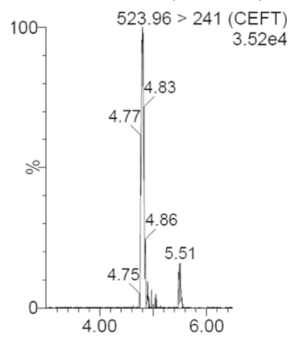

Ceftioflur (CEFT)

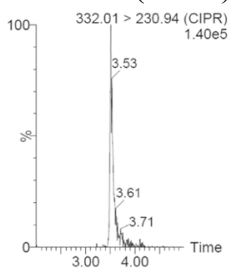

Ciprofloxacin (CIPR)

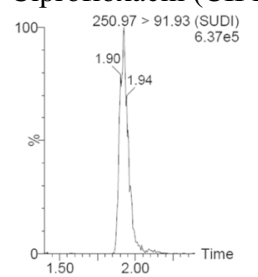

Sulfadiazin (SUDI)

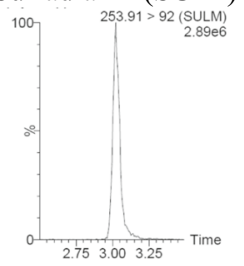

Sulfamethoxazol (SULM)

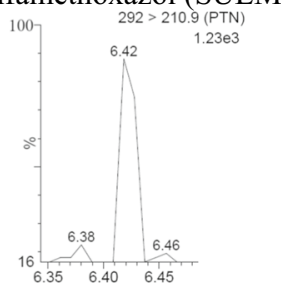

Parathion (PTN)

Fig. 1 continued 


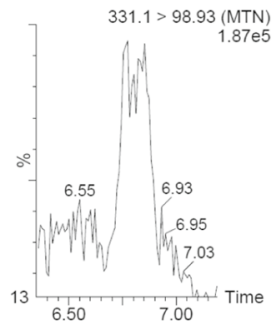

Malathion (MTN)
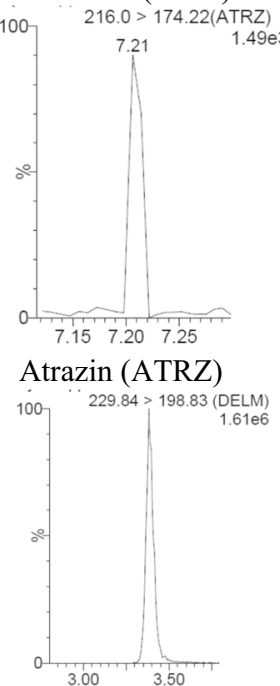

Deltametrin (DELM)

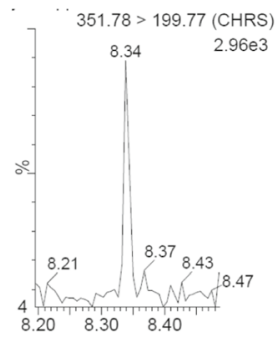

Chloropyriphos (CHRS)

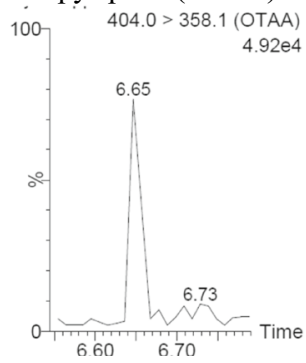

Ochratoxin A (OTAA)

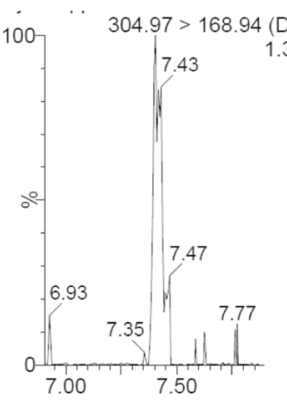

Diazinon (DNN)

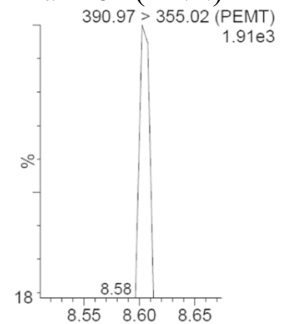

\section{Permetrin (PEMT)}

${ }^{100} \quad 362.9>306.86(\mathrm{COU})$

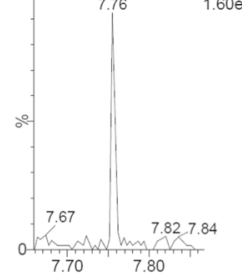

Coumaphos (COU)

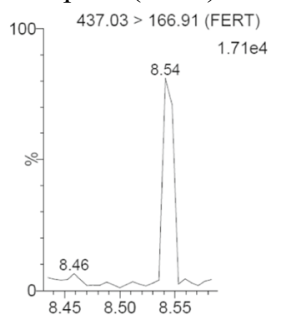

Fenvalerat (FERT)

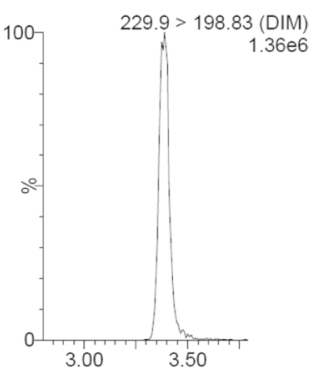

Dimethoat (DIM)

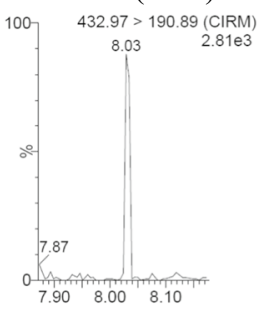

\section{Cypermetrin (CIRM)}

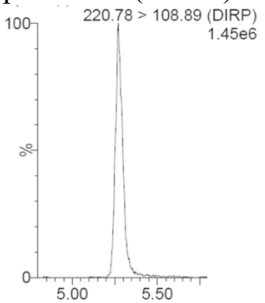

Dichlorphos (DIRP)

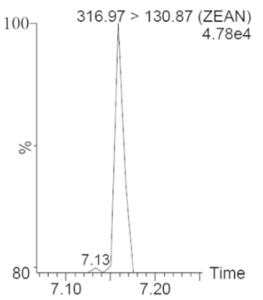

Zearalenon (ZEAN)

Fig. 1 continued 


\begin{abstract}
Abbreviations
CCa: Decision limit; CCß: Detection capability; LOD: Limit of detection; LOQ: Limit of quantification; CV: Coefficient of variation; LC-MS/MS: Liquid chromatography tandem mass spectrometry; SPE: Solid-phase extraction; QuEChERS: Quick, easy, cheap, effective, rugged, and safe; MeCN: Acetronitrile; $\mathrm{MeOH}$ : Methanol; MRL: Maximum recommended residue levels; MRPL: Minimum required performance limits; EtOAc: Ethylacetate; MRM: Multiple reaction monitoring; CRMs: Certified reference materials; ESI: Electrospray ionisation; $\mathrm{S} / \mathrm{N}$ : Signal to noise ratio; $\mathrm{R}^{2}$ : Coefficient of corelation.
\end{abstract}

\section{Supplementary Information}

The online version contains supplementary material available at https://doi. org/10.1186/s13065-021-00788-5.

Additional file 1. Recoveries from investigation of sample amount and extraction steps.

Additional file 2. Chromatograms of positive samples from bovine meat.

\section{Acknowledgements}

Not applicable.

\section{Authors' contributions}

ZHM: participating in writing the maniscrupt, experimental desigin, methodology, data analysis, review and editing resources, project administration and supervision. RU participating in writing the maniscrupt, experimental desigin, methodology, sample preparation, analysis and data analysis. SJ carried out sampling, sample preparation, analysis and data analysis. DJ, VS and LP participating in conceptualization and investigation. JJS participated in project administration, funding acquisition, review and editing. All authors read and approved the final manuscript.

\section{Funding}

This work was supported by the IAEA project Integrated radiometric and complementary techniques for mixed contaminants and residues in food (D52041).

\section{Availability of data and materials}

All data and materials analyzed during the current study are included in this manuscript.

\section{Declarations}

Ethics approval and consent to participate.

Not applicable.

\section{Consent for publication}

Not applicable.

\section{Competing interests}

The authors of the present work declare that they have no competing interests.

\section{Author details}

${ }^{1}$ Faculty of Veterinary Medicine-Skopje, University "Ss. Cyril and Methodius", Lazar Pop-Trajkov 5/7, 1000 Skopje, Republic of North Macedonia. ${ }^{2}$ International Atomic Energy Agency, Vienna International Centre, P. O. Box 100, 1400 Vienna, Austria.

Received: 12 June 2020 Accepted: 23 November 2021

Published online: 08 December 2021

\section{References}

1. Anastassiades M, Lehotay SJ, Stajnbaher D, Schenk FJ. Fast and easy multiresidue method employing acetonitrile extraction/partitioning and "dispersive solid-phase extraction" for the determination of pesticide residues in produce. J AOAC Int. 2003;86(2):412-31.

2. Aguilera-Luiz MM, Martínez Vidal JL, Romero-González R, Garrido FA. Multiresidue determination of veterinary drugs in milk by ultra-high pressure liquid chromatography tandem mass spectrometry. J Chromatogr A. 2008. https://doi.org/10.1016/j.chroma.2008.07.066.

3. Biselli S, Schwalb U, Meyer A, Hartig L. A multi-class, multi-analyte method for routine analysis of 84 veterinary drugs in chicken muscle using simple extraction and LC-MS/MS. Food Addit Contam Part A. 2013. https://doi.org/10.1080/19440049.2013.777976.

4. Bitas D, Kabir A, Locatelli M, Samanidou V. Food sample preparation for the determination of sulfonamides by high-performance liquid chromatography: state-of-the-art. Separations. 2018. https://doi.org/10.3390/ separations5020031.

5. Danezis GP, Anagnostopoulos CJ, Liapis K, Koupparis MA. Multi-residue analysis of pesticides, plant hormones, veterinary drugs and mycotoxins using HILIC chromatography-MS/MS in various food matrices. Anal Chim Acta. 2016. https://doi.org/10.1016/j.aca.2016.09.011.

6. European Commission. Decision (2002/657/EC) of 12 August 2002 implementing Council Directive 96/23/EC concerning the performance of analytical methods and interpretation of results. OJEU, L221, 2002. 8-36.

7. European Commision. Commision Reguation (EU) No. 1881/2006 of 19 December 2006: setting maximum levels for certain contaminants in foodstuffs. OJEU, L364/5, 2006. 1-20.

8. European Commission. Commission Regulation (EU) No. 37/2010 of 22 December 2009: on pharmacologically active substances and their classification regarding maximum residue limits in foodstuffs of animal origin. OJEU, L15, 2010. 1-72.

9. European Commission. SANTE/12682/2019. Guidance document on analytical quality control and method validation procedures for pesticide residues analysis in food and feed. 2019.

10. EU pesticide database. https://ec.europa.eu/food/plant/pesticides/eupesticides-database/public/?event=pesticide.residue.selection\&langu age $=$ EN.

11. Gómez-Pérez ML, Romero-González R, Vidal JLM, Frenich AG. Analysis of pesticide and veterinary drug residues in baby food by liquid chromatography coupled to Orbitrap high resolution mass spectrometry. Talanta. 2015. https://doi.org/10.1016/j.talanta.2014.07.066.

12. Kaufmann A, Butcher P, Maden K, Widmer M. Quantitative multiresidue method for about 100 veterinary drugs in different meat matrices by sub 2- $\mu \mathrm{m}$ particulate high-performance liquid chromatography coupled to time of flight mass spectrometry. J Chromatogr A. 2008 https://doi.org/10.1016/j.chroma.2008.03.089.

13. Marazuela MD. Determination of veterinary drug residues in foods by liquid chromatography-mass spectrometry: basic and cutting-edge applications. In: Fanali S, Haddad PR, Poole C, Riekkola ML, editors. Liquid chromatography: applications. 2nd ed. Amsterdam: Elsevier; 2017. p. 539-70.

14. Reig M, Toldrá F. Veterinary drug residues in meat: concerns and rapid methods for detection. Meat Sci. 2008. https://doi.org/10.1016/j.meats ci.2007.07.029.

15. Ren Y, Zhang Y, Shao S, Cai Z, Feng L, Pan H, Wang Z. Simultaneous determination of multi-component mycotoxin contaminants in foods and feeds by ultra-performance liquid chromatography-tandem mass spectrometry. J Chromatogr A. 2007. https://doi.org/10.1016/j.chroma. 2006.12.064

16. SANCO. CRLs view on state of the art analytical methods for national residue control plans. CRL Guidance Paper (7 December 2007) 2007. pp. 1-8.

17. Stubbings $G$, Bigwood T. The development and validation of a multiclass liquid chromatography tandem mass spectrometry procedure for the determination of veterinary drug residues in animal tissue using a QuEChERS (QUick, Easy, CHeap, Effective, Rugged and Safe) approach. Anal Chim Acta. 2009. https://doi.org/10.1016/j.aca.2009.01.029.

18. Villar-Pulido M, Gilbert-López B, García-Reyes JF, Ramos-Martos N, Molina-Díaz A. Multiclass detection and quantitation of antibiotics and veterinary drugs in shrimps by fast liquid chromatography time-offlight mass spectrometry. Talanta. 2011. https://doi.org/10.1016/j.talan ta.2011.06.036

19. Wei H, Tao Y, Chen D, Xie S, Pan Y, Liu Z, Huang L, Yuan Z. Development and validation of a multi-residue screening method for veterinary 
drugs, their metabolites and pesticides in meat using liquid chromatography-tandem mass spectrometry. Food Addit Contam Part A. 2015. https://doi.org/10.1080/19440049.2015.1008588.

20. Whelan M, Kinsella B, Furey A, Moloney M, Cantwell H, Letohay SJ, Danaher M. Determination of anthelmintic drug residues in milk using ultra high-performance liquid chromatography-tandem mass spectrometry with rapid polarity switching. J Chromatogr A. 2010. https://doi.org/10. 1016/j.chroma.2010.05.007.

21. Xie J, Peng T, Zhu A, He J, Chang Q, Hu X, Chen H, Fan C, Jiang W, Chen M, $\mathrm{Li}$, Ding $\mathrm{S}$, Jiang $\mathrm{H}$. Multi-residue analysis of veterinary drugs, pesticides and mycotoxinsin dairy products by liquid chromatography-tandem mass spectrometry using low-temperature cleanup and solid phase extraction. J Chromatogr B Analyt Technol Biomed Life Sci. 2015. https:// doi.org/10.1016/j.jchromb.2015.08.005.

22. Yamada R, Kozono M, Ohmori T, Morimatsu F, Kitayama M. Simultaneous determination of residual veterinary drugs in bovine, porcine, and chicken muscle using liquid chromatography coupled with electrospray ionization tandem mass spectrometry. Biosci Biotechnol Biochem. 2006. https://doi.org/10.1271/bbb.70.54.

23. Zhan J, Xu DM, Wang SJ, Sun J, Xu YJ, Ni ML, Yin JY, Chen J, Yu XJ, Huang ZQ. Comprehensive screening for multi-class veterinary drug residues andother contaminants in muscle using column-switching UPLC-MS/MS. Food Addit Contam Part A. 2013. https://doi.org/10.1080/19440049.2013. 833670.

24. Zhan J, Zhong YY, Yu XJ, Peng JF, Chen SB, Yin JY, Zhang JJ, Zhu Y. Multiclass method 24 for determination of veterinary drug residues and other contaminants in infant formula by ultra performance liquid chromatographytandem mass spectrometry. Food Chem. 2013. https://doi.org/10. 1016/j.foodchem.2012.09.130

\section{Publisher's Note}

Springer Nature remains neutral with regard to jurisdictional claims in published maps and institutional affiliations.

- fast, convenient online submission

- thorough peer review by experienced researchers in your field

- rapid publication on acceptance

- support for research data, including large and complex data types

- gold Open Access which fosters wider collaboration and increased citations

- maximum visibility for your research: over $100 \mathrm{M}$ website views per year

At BMC, research is always in progress.

Learn more biomedcentral.com/submissions 